\title{
Parameter estimation of inspiralling compact binaries using 3.5 post-Newtonian gravitational wave phasing: The non-spinning case
}

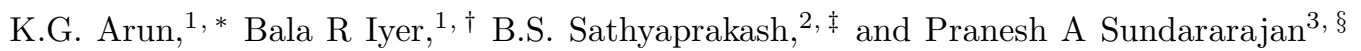 \\ ${ }^{1}$ Raman Research Institute, Bangalore 560 080, India \\ ${ }^{2}$ School of Physics and Astronomy, Cardiff University, 5, The Parade, Cardiff, UK, CF24 3YB \\ ${ }^{3}$ Birla Institute of Technology and Science, Pilani
}

(Dated: October 22, 2018)

\begin{abstract}
We revisit the problem of parameter estimation of gravitational-wave chirp signals from inspiralling non-spinning compact binaries in the light of the recent extension of the post-Newtonian $(\mathrm{PN})$ phasing formula to order $(v / c)^{7}$ beyond the leading Newtonian order. We study in detail the implications of higher post-Newtonian orders from $1 \mathrm{PN}$ up to $3.5 \mathrm{PN}$ in steps of $0.5 \mathrm{PN}(\sim v / c)$, and examine their convergence. In both initial and advanced detectors the estimation of the chirp mass $(\mathcal{M})$ and symmetric mass ratio $(\eta)$ improve at higher PN orders but oscillate with every half-a-PN order. In initial LIGO, for a $10 M_{\odot}-10 M_{\odot}$ binary at a signal-to-noise ratio (SNR) of 10, the improvement in the estimation of $\mathcal{M}(\eta)$ at $3.5 \mathrm{PN}$ relative to $2 \mathrm{PN}$ is $\sim 19 \%(52 \%)$. We compare parameter estimation in different detectors and assess their relative performance in two different ways: at a fixed $S N R$, with the aim of understanding how the bandwidth improves parameter estimation, and for a fixed source, to gauge the importance of sensitivity. Errors in parameter estimation at a fixed SNR are smaller for VIRGO than for both initial and advanced LIGO. This is because of the larger bandwidth over which it observes the signals. However, for sources at a fixed distance it is advanced LIGO that achieves the lowest errors owing to its greater sensitivity. Finally, we compute the amplitude corrections due to the 'frequency-sweep' in the Fourier domain representation of the waveform within the stationary phase approximation and discuss its implication on parameter estimation. We find that the amplitude corrections change the errors in $\mathcal{M}$ and $\eta$ by less than $10 \%$ for initial LIGO at a signal-to-noise ratio of 10. Our analysis makes explicit the significance of higher PN order modelling of the inspiralling compact binary on parameter estimation.
\end{abstract}

PACS numbers: $04.25 \mathrm{Nx}, 04.30,04.80 . \mathrm{Nn}, 97.60 . \mathrm{Jd}, 95.55 \mathrm{Ym}$

\section{INTRODUCTION}

With the advent of a new generation of gravitational wave (GW) detectors such as LIGO, VIRGO, GEO and TAMA 1], we are on the eve of a new era in astronomy: Gravitational Wave Astronomy (see Ref. 2, 3] for recent reviews). The paucity of GW sources within a detectable distance, as well as the weakness of the gravitational wave signals, make imperative the necessity for developing optimal data analysis techniques, both for their detection and for the extraction of maximum information from these signals. It is for this reason that inspiralling compact binaries, which can be well modelled within the general relativistic framework, have become one of the most promising candidate sources for the large and medium scale gravitational wave detectors.

An efficient data analysis scheme involves two independent aspects: first, the theoretical computation of very high accuracy templates and second, the design of a detection strategy adapted to the particular signal one is looking for. These strategies vary according to the type of signal. Gravitational waves from inspiralling binaries are transients lasting for a short duration in the sensitivity bandwidth of a ground-based detector. As the binary evolves the waveform sweeps up in frequency and amplitude, leading to a characteristic chirp signal. As the phasing of the waves is known very accurately, it is possible to enhance their detectability by using matched filtering. Bursts of unknown shape, as for example from a supernova, will be probed by monitoring the power excesses in the Fourier or time-frequency domain, but the enhancement in the visibility of the signal is not as good as when the phasing of the signal is known and matched filtering can be applied. In both cases, coincident observations with a network of detectors would assist the detection significantly, by increasing the confidence level of detection and mitigating nonstationarity. Continuous sinusoidal signals, as for example from a spinning neutron star, are also detected by matched

\footnotetext{
${ }^{*}$ Electronic address: arun AT rri.res.in

${ }^{\dagger}$ Electronic address: bri AT rri.res.in

${ }^{\ddagger}$ Electronic address: B.Sathyaprakash AT astro.cf.ac.uk

$\S$ Electronic address: pranesh AT gmail.com
} 
filtering and the signal visibility increases as the square-root of the period for which the signal is observed. Stochastic signals require cross-correlation of data from two or more collocated, or geographically close by, detectors. Here, the stochastic signal buried in one of the instruments acts as a matched filter to dig out exactly (or nearly exactly) the same signal in another. However, since the filter is noisy the efficiency is greatly degraded and the visibility improves only as the fourth-root of the duration of observation.

As a binary inspirals adiabatically, i.e. when the inspiral time-scale is much larger than the orbital time-scale, it is possible to treat the problem perturbatively and expand the general relativistic equations of motion and wave generation as a power series in $v / c$, where $v$ is the characteristic orbital velocity of the system. This post-Newtonian $(\mathrm{PN})$ treatment has been successful in modelling the dynamics of a binary even at the late stages of inspiral and used in the computation of waveforms necessary for data analysis (see [4] for a recent review) ${ }^{1}$. Since radiation back reaction causes the orbital eccentricity $e$ to fall-off, for small $e$, as $e \propto P^{19 / 18}$ and the orbital radius to decay much more slowly $r \propto P^{2 / 3}[5]$, the binary orbit will essentially be circular by the time the system reaches the late stages of the inspiral phase. Thus, in our analysis we shall restrict our attention to the case of compact binaries in quasi-circular orbit, i.e. circular but for the adiabatic decay of the orbit under gravitational radiation reaction.

\section{A. Data analysis of the chirp signal: Matched filtering}

Among the different methods suggested for the detection of chirps from inspiralling and merging binaries, matched filtering (also known as Weiner filtering) is the most effective technique [6, 7, 8]. Matched filtering consists of passing the detector data through a linear filter, or a template, constructed from the expected signal $h(t ; \boldsymbol{\theta})$. Here $\boldsymbol{\theta}$ is a 'vector' whose components are the parameters of the template. The templates $h(t ; \boldsymbol{\theta})$ generally use the restricted waveform where for binaries in quasi-circular orbits the phase is computed at the highest PN order available, but the amplitude is taken to be Newtonian, involving only the dominant signal harmonic at twice the orbital frequency. This is different from the complete waveform, which incorporates the PN corrections to the amplitude, arising from the 'plus' and 'cross' GW polarizations, and hence includes the contribution from other harmonics (both higher and lower) besides the dominant one. Till date, for non-spinning binaries, the restricted waveform is computed to 3.5PN accuracy [9, 10] and the complete waveform up to $2.5 \mathrm{PN}$ order [11, 12]. The best template is probably the one which consists of the phasing at 3.5PN and the amplitude at 2.5PN. Presently, both the detection and parameter estimation problems mainly employ the restricted $\mathrm{PN}$ waveform although there have been some investigations on the ensuing improvement achieved when corrections arising from the other harmonics are incorporated by using the complete waveform [13, 14, 15]. In this paper, we confine ourselves mostly to the restricted waveform; specific amplitude corrections arising from the 'frequency-sweep' are considered, however, in Sec. IV]

In matched filtering, the unknown set of parameters characterizing the signal are measured by maximising the correlation of the data with a whole family of templates which correspond to different values of the parameters. The parameters of the template which maximises the output of a matched filter is an estimate of the true parameters. The parameters of a signal measured in a single experiment will be different from the actual values due to the presence of noise. Parameter estimation basically aims at computing the probability distribution for the measured values of a signal. Given a measured value from a single experiment one then uses the probability distribution function to compute the interval in which the true parameters of the signal lie at a specified confidence level (see Sec. II for a summary of the theory of parameter estimation). In the next Section, we discuss the types of error bounds proposed in the literature in the context of GW data analysis.

\section{B. Parameter estimation of chirp signal: Different kinds of error bounds}

In parameter estimation it is of interest to obtain the distribution of the measured values and error bounds on the measured values of the parameters. To this end, the starting point would be to construct the Fisher information matrix, the inverse of which, the covariance matrix, provides an estimate of the possible errors in the measurement of the parameters [7]. Error bounds obtained using the covariance matrix are called the Cramer-Rao bounds [16]. However, for low values of the signal-to-noise ratio (SNR) the actual errors involved may be much larger than the errors estimated by this method. Cramer-Rao bounds fall off as the inverse of SNR, whereas the actual errors need not follow this behaviour. One usefulness of the Cramer-Rao bound is that, they are asymptotically valid in the limit of high SNR and hence provides a basis to test all other estimates.

\footnotetext{
${ }^{1}$ In our nomenclature, $(v / c)^{n}$ corresponds to $\frac{n}{2}$ post-Newtonian $(\mathrm{PN})$ order. Henceforth, we shall use units in which $c=G=1$.
} 
An alternate, and more general, way is to estimate the errors by Monte Carlo methods [17, 18, 19]. In this method, one mimics the detection problem on a computer by performing a large number of simulations corresponding to different realizations of the noise in each one of them. The advantage here is that, one no longer assumes a high SNR, which is a crucial assumption in computing the covariance matrix. In Ref. [18] exhaustive Monte Carlo simulations were carried out to compute the errors in the estimation of the parameters and the covariances among them. It used the initial LIGO configuration and took into account only the $1 \mathrm{PN}$ corrections assuming, as usual, the orbit to be quasi-circular. It was shown that the covariance matrix grossly underestimates the errors in the estimation of the parameters by over a factor of two at a SNR of 10. This discrepancy disappears when the SNR is approximately 15 for a Newtonian filter and 25 for the 1PN case. Further, the reason for the discrepancy was explained in detail in Ref. [20]. Extending the Monte Carlo simulations of Ref. [18] by the inclusion of higher order terms would be computationally quite expensive [20].

More rigorous bounds (Weiss-Weinstein bound and Ziv-Zakai bound) on the parameter estimation of inspiralling binaries are discussed in Ref. [21]. They compare, at the Newtonian order, the results obtained by these bounds with the Cramer-Rao bounds and the numerical Monte Carlo results. At large SNR, they find all theoretical bounds to be identical and attained by Monte Carlo methods. At SNRs below 10, the Weiss-Weinstein bound and the Ziv-Zakai bound provide increasingly tighter lower bounds than the Cramer-Rao bound.

\section{Parameter estimation and the phasing formula: An update}

Intrinsic parameters, like masses and spins, characterising the signal can be estimated from the data collected by a single detector. On the other hand, the distance to the source and its position in the sky require at least three geographically separated detectors forming a detector network [22, 23, 24]. Cutler and Flanagan [22] have shown that, to a good approximation, it is sufficient to use Newtonian waveforms in these analyses. We will not, however, concern ourselves with the estimation of distance in the present work.

Cutler and Flanagan [22] initiated the study of the implications of higher order phasing formula as applied to the parameter estimation of inspiralling binaries. They used the 1.5PN phasing formula to investigate the problem of parameter estimation, both for spinning and non-spinning binaries, and examined the effect of the spin-orbit parameter $\beta$ (assumed constant) on the estimation of parameters. They find that parameter estimation worsens by a factor of about ten because of the inclusion of $\beta$. The effect of the $2 \mathrm{PN}$ phasing formula was analysed independently by Poisson and Will 25] and Królak, Kokkotas and Schäfer [26]. In both of these works the focus was to understand the new spin-spin coupling term $\sigma$ appearing at the second PN order when the spins were aligned perpendicular to the orbital plane (constant $\beta$ and $\sigma$ ). Compared to Ref. [26], Ref. [25] also included the a priori information about the magnitude of the spin parameters, which then leads to a reduction in the rms errors in the estimation of mass parameters. It was shown that the effect of the inclusion of $\sigma$ is less drastic than $\beta$ and that it worsens parameter estimation only by a factor of order unity. In a more recent work 27], the implications of including the spin couplings on the parameter estimation and the tests of alternative theories of gravity were studied using the LISA noise curve.

\section{Summary of the current work}

Starting with a brief summary of parameter estimation in Sec. III we discuss in Sec. IIIA the nature of the 'chirp' signals from non-spinning binaries using the $3.5 \mathrm{PN}$ phasing formula 10 which is now completely determined following the recent computation of the hitherto unknown parameters at 3PN 28, 29, 30, 31, 32, 33, 34].

We study parameter estimation using three different noise curves: advanced LIGO, initial LIGO and VIRGO. Our choice is motivated by the fact that initial LIGO and VIRGO are the more sensitive instruments among the first generation of interferometric detectors with a somewhat different combination of bandwidth and sensitivity while advanced LIGO is prototypical of second generation instruments currently being planned. We will use the planned design sensitivity curves of initial LIGO and VIRGO as in Ref. [35] and advanced LIGO ${ }^{2}$ as in Ref. [2] and discuss in Sec. IIIB the sensitivity and span of these instruments for binary coalescences.

As mentioned earlier, Poisson and Will [25] analysed the implications of the 2PN phasing formula on parameter estimation of spinning binaries [36]. However, extending this to higher orders is not possible at present since spin effects beyond $2 \mathrm{PN}$ have not yet been computed. Therefore, in this work we will follow the procedure adopted in

\footnotetext{
${ }^{2}$ For the sake of comparison with previous work we have also carried out our study with the advanced LIGO noise curve as in Refs. [22, 25]. However, most of the work reported in this study uses the advanced LIGO noise curve quoted in Ref. [2].
} 
[25], but consider only the non-spinning case. We study in Sec. IIIC] the effect of higher order phasing terms by incorporating them in steps of half-a-PN order from $1 \mathrm{PN}$ up to $3.5 \mathrm{PN}$ and examine the convergence of parameter estimation with PN orders. We compare the errors for the different noise curves and assess their relative performance in two different ways: at a fixed signal-to-noise ratio (Sec. IIIC), with the aim of understanding how the sensitivity bandwidth improves parameter estimation, and for a fixed source (Sec. IIID), to gauge the relative importance of sensitivity and bandwidth. We have examined the correlation of parameter estimation results to the number of useful cycles [37] and the sensitivity bandwidth (Sec. [IIE), which together can explain the performance of different detectors with regard to parameter estimation.

In Sec. IV we study the effect of the amplitude terms arising from the 'frequency-sweep' $d F / d t$ within the stationary phase approximation [38]. These corrections cause the SNR (which is related to the total energy emitted by the system) of a given binary to vary as we go from lower to higher PN orders. The results are compared against the standard restricted waveform approach and should be viewed as a prelude, albeit inconsistent, to parameter estimation using the complete waveform. We conclude in Sec. $\mathbf{\nabla}$ with a summary of our results, their regime of validity, limitations and future directions.

Our main conclusion is that the 3.5PN phasing formula leads to an improved estimate of the binary parameters. For instance, in the case of black hole binaries, at a SNR of 10, the estimate of chirp mass (symmetric mass ratio), more specifically $\ln \mathcal{M}(\ln \eta)$, improves while using the $3.5 \mathrm{PN}$ phasing formula as compared to the $2 \mathrm{PN}$ by about $19 \%(52 \%)$. Improvements are seen in all cases but are relatively smaller for lighter binaries. At a fixed SNR, VIRGO provides a better estimate of the parameters compared to both initial and advanced LIGO configurations owing to its better sensitivity bandwidth. This is true over the entire mass range and even for lower mass binaries for which VIRGO accumulates fewer number of useful cycles. For a fixed source, however, advanced LIGO measures the parameters most accurately, as expected, with VIRGO doing better than initial LIGO. Our investigation of the amplitude corrections from 'frequency-sweep' within the stationary phase approximation finds that the percentage change induced by this effect in parameter estimation is less than $10 \%$ for initial LIGO at a SNR of 10 .

\section{A BRIEF SUMMARY OF PARAMETER ESTIMATION THEORY}

A firm statistical foundation to the theory of gravitational wave data analysis was laid down by the works of e.g. Finn and Chernoff [39, 40] and Cutler and Flanagan [22]. This Section briefly outlines the problem of parameter estimation relevant to this paper. Notation and treatment of this Section essentially follow Ref. [22, 25, 41] (see also [7, 42, 43, 44] for further details). We restrict our discussion to measurements made by a single detector.

\section{A. Matched filtering}

The output of a gravitational wave detector contains both the signal and noise and is schematically represented as

$$
x(t)=h(t)+n(t),
$$

where $x(t)$ is the signal registered and $n(t)$ is the noise, which is assumed to be a stationary Gaussian random variable, with zero mean, i.e.,

$$
\overline{n(t)}=0 \text {. }
$$

Here an overbar denotes the ensemble average (over many realisations of the noise or, equivalently, over an ensemble of detectors). Let $q(t)$ define a linear filter and $c(t)$ its correlation with the detector output $x(t)$

$$
c(t)=\int_{-\infty}^{\infty} d t^{\prime} x\left(t^{\prime}\right) q\left(t+t^{\prime}\right) .
$$

Define a new quantity $\sigma[q](t)$, such that $c(t)$ is normalized by the square root of its variance,

$$
\sigma[q](t)=\frac{c(t)}{\left[\overline{c^{2}(t)}-\overline{c(t)}^{2}\right]^{1 / 2}}=\frac{2 \Re \int_{0}^{\infty} d f \tilde{x}(f) \tilde{q}^{*}(f) e^{2 \pi i f t}}{\left[\int_{0}^{\infty} d f S_{h}(f)|\tilde{q}(f)|^{2}\right]^{1 / 2}},
$$

where $\tilde{x}(f)$ and $\tilde{q}(f)$ are the Fourier transforms of $x(t)$ and $q(t)$, respectively, $S_{h}(f)$ is the real, one-sided power spectral density defined only for positive frequencies by

$$
\overline{n(f) \tilde{n}^{*}\left(f^{\prime}\right)}=\frac{1}{2} \delta\left(f-f^{\prime}\right) S_{h}(f),
$$


and $\tilde{n}(f)$ is the Fourier transform of $n(t)$ defined as $\tilde{n}(f)=\int_{-\infty}^{\infty} d t n(t) e^{-2 \pi i f t}$. The filtered SNR is defined by the ensemble average

$$
\rho[q](t)=\overline{\sigma[q](t)}=\frac{2 \Re \int_{0}^{\infty} d f \tilde{h}(f) \tilde{q}^{*}(f) e^{2 \pi i f t}}{\left[\int_{0}^{\infty} d f S_{h}(f)|\tilde{q}(f)|^{2}\right]^{1 / 2}} .
$$

An optimal filter is the one which maximises the SNR at a particular instant, say $t=0$, and is given by the matched filtering theorem as

$$
\tilde{q}(f)=\gamma \frac{\tilde{h}(f)}{S_{h}(f)},
$$

where $\gamma$ is an arbitrary real constant. Thus, the SNR corresponding to the optimal filter is given by

$$
\rho^{2}=4 \int_{0}^{\infty} d f \frac{|\tilde{h}(f)|^{2}}{S_{h}(f)}
$$

\section{B. Parameter estimation}

Though we may have a prior knowledge of the form of the signal we will not know what its parameters are. Indeed, the parameters are to be measured in the process of matched filtering. This is achieved by maximising the correlation in Eq. (2.4) with a whole family of templates corresponding to different values of the signal parameters. The parameters of the filter which maximise the correlation are the measured values attributed by the analyst to the signal presumed to be buried in the data. These parameters need not agree, in general, with the actual parameters of the signal since the measured values depend on a particular realization of the detector noise.

For a given incident gravitational wave, different realizations of the noise will give rise to somewhat different best-fit parameters. However, if the SNR is high enough, the best-fit parameters will have a Gaussian distribution centered around the actual values.

Let $\tilde{\theta}^{a}$ denote the 'true values' of the parameters and let $\tilde{\theta}^{a}+\Delta \theta^{a}$ be the best-fit parameters in the presence of some realization of the noise. Then for large SNR, errors in the estimation of parameters $\Delta \theta^{a}$ obey a Gaussian probability distribution of the form 39

$$
p\left(\Delta \theta^{a}\right)=p^{(0)} e^{-\frac{1}{2} \Gamma_{b c} \Delta \theta^{b} \Delta \theta^{c}},
$$

where $p^{(0)}$ is a normalization constant. In the above expression $\Gamma_{a b} \equiv\left(h_{a} \mid h_{b}\right)$, where $h_{a} \equiv \partial h / \partial \theta^{a}$, is the Fisher information matrix evaluated at the measured value $\hat{\theta}$ of the parameters $\theta$. Here, $(\mid)$ denotes the noise weighted inner product. Given any two functions $g$ and $h$ their inner product is defined as:

$$
(g \mid h) \equiv 2 \int_{0}^{\infty} d f \frac{\tilde{g}^{*}(f) \tilde{h}(f)+\tilde{g}(f) \tilde{h}^{*}(f)}{S_{h}(f)} .
$$

Using the definition of the inner product one can re-express $\Gamma_{a b}$ more explicitly as

$$
\Gamma_{a b}=2 \int_{0}^{\infty} \frac{\tilde{h}_{a}^{*}(f) \tilde{h}_{b}(f)+\tilde{h}_{a}(f) \tilde{h}_{b}^{*}(f)}{S_{h}(f)} d f .
$$

The variance-covariance matrix, or simply the covariance matrix, defined as the inverse of the Fisher information matrix, is given by

$$
\Sigma^{a b} \equiv\left\langle\Delta \theta^{a} \Delta \theta^{b}\right\rangle=\left(\Gamma^{-1}\right)^{a b},
$$

where $\langle\cdot\rangle$ denotes an average over the probability distribution function in Eq. (2.9). The root-mean-square error $\sigma_{a}$ in the estimation of the parameters $\theta^{a}$ is

$$
\sigma_{a}=\left\langle\left(\Delta \theta^{a}\right)^{2}\right\rangle^{1 / 2}=\sqrt{\Sigma^{a a}}
$$

and the correlation coefficient $c^{a b}$ between parameters $\theta^{a}$ and $\theta^{b}$ is defined as

$$
c^{a b}=\frac{\left\langle\Delta \theta^{a} \Delta \theta^{b}\right\rangle}{\sigma_{a} \sigma_{b}}=\frac{\Sigma^{a b}}{\sqrt{\Sigma^{a a} \Sigma^{b b}}} .
$$


(There is no summation over repeated indices in Eqs. (2.13) and (2.14).) As a consequence of their definition the correlation coefficients must lie in the range $[-1,1]$. When the correlation coefficient between two parameters is close to 1 (or -1 ), it indicates that the two parameters are perfectly correlated (respectively, anti-correlated) (and therefore redundant) and a value close to 0 indicates that the two parameters are uncorrelated; covariance close to 1 (or -1) among parameters causes a large dispersion in their measurement.

In our analysis we will apply the method outlined above to three prototypical systems normally considered in gravitational wave studies related to ground-based detectors. These include a binary neutron star system (NS-NS), a neutron star-black hole system (NS-BH) and a binary black hole system (BH-BH). Throughout our analysis we shall assume that the mass of a NS is $1.4 M_{\odot}$ and that of a $\mathrm{BH}$ is $10 M_{\odot}$.

\section{PARAMETER ESTIMATION USING THE 3.5PN PHASING FORMULA}

Having outlined the essential results from the theory of parameter estimation, we proceed to address the question of extracting the parameters from the chirp signal using the 3.5PN phasing formula. Our computation parallels the one by Poisson and Will [25] except that we confine our attention to the case of non-spinning binaries whereas Ref. [25] dealt with spinning binaries.

\section{A. Fourier transform of chirp at 3.5PN order}

To compute the Fisher information matrix we would need the Fourier transform $\tilde{h}(f)$ of the signal $h(t)$. (Note that here and below $f$ is the Fourier transform variable which should not be confused with $F$, the instantaneous frequency of emitted radiation.) Following earlier works, we employ the stationary phase approximation (SPA) to evaluate the Fourier amplitude of the waveform. Given a function $B(t)=2 A(t) \cos \phi(t)$, where $d \ln A / d t \ll d \phi(t) / d t$ and $\left|d^{2} \phi / d t^{2}\right| \ll(d \phi / d t)^{2}$, the SPA provides the following estimate of the Fourier transform $\tilde{B}(f)$ :

$$
\tilde{B}(f) \simeq \frac{A\left(t_{f}\right)}{\sqrt{\dot{F}\left(t_{f}\right)}} e^{i\left[\Psi_{f}\left(t_{f}\right)-\frac{\pi}{4}\right]}, f \geq 0,
$$

where $\Psi_{f}(t) \equiv 2 \pi f t-\phi(t)$,

$$
\text { and } \frac{d \phi}{d t} \equiv 2 \pi F(t)
$$

In this equation $t_{f}$ is defined as the time at which $F\left(t_{f}\right)=f$ and $\Psi_{f}\left(t_{f}\right)$ is the value of $\Psi_{f}(t)$ at $t=t_{f}$. Starting from the 3.5PN phasing formula in [10], the Fourier transform has been explicitly calculated in Refs. [35, 45]. This Fourier domain waveform, which forms the basis of our further calculations, is given by

$$
\tilde{h}(f)=\mathcal{A} f^{-7 / 6} e^{i \psi(f)},
$$

where $\mathcal{A} \propto \mathcal{M}^{5 / 6} Q$ (angles) $/ D$, and to $3.5 \mathrm{PN}$ order the phase of the Fourier domain waveform is given by

$$
\begin{aligned}
\psi(f) & \equiv \Psi_{f}\left(t_{f}\right)-\frac{\pi}{4} \\
& =2 \pi f t_{c}-\phi_{c}-\frac{\pi}{4}+\frac{3}{128 \eta v^{5}} \sum_{k=0}^{N} \alpha_{k} v^{k},
\end{aligned}
$$

where $v=(\pi M f)^{1 / 3}, M=m_{1}+m_{2}$ is the total mass of the binary, $\eta=m_{1} m_{2} / M^{2}$ is the dimensionless mass ratio and $D$ the distance to the binary. We shall find it useful in our study to deal with the chirp mass defined by $\mathcal{M}=\eta^{3 / 5} M$ rather than the total mass $M$. The coefficients $\alpha_{k}$ 's, $k=0, \ldots, N$, (with $N=7$ at $3.5 \mathrm{PN}$ order) in the Fourier phase 
are given by

$$
\begin{aligned}
\alpha_{0} & =1 \\
\alpha_{1} & =0 \\
\alpha_{2} & =\frac{20}{9}\left(\frac{743}{336}+\frac{11}{4} \eta\right), \\
\alpha_{3} & =-16 \pi \\
\alpha_{4} & =10\left(\frac{3058673}{1016064}+\frac{5429}{1008} \eta+\frac{617}{144} \eta^{2}\right), \\
\alpha_{5} & =\pi\left(\frac{38645}{756}+\frac{38645}{252} \log \left(\frac{v}{v_{\text {lso }}}\right)-\frac{65}{9} \eta\left[1+3 \log \left(\frac{v}{v_{\text {lso }}}\right)\right]\right), \\
\alpha_{6} & =\left(\frac{11583231236531}{4694215680}-\frac{640 \pi^{2}}{3}-\frac{6848 \gamma}{21}\right)+\eta\left(-\frac{15335597827}{3048192}+\frac{2255 \pi^{2}}{12}-\frac{1760 \theta}{3}+\frac{12320 \lambda}{9}\right) \\
& +\frac{76055}{1728} \eta^{2}-\frac{127825}{1296} \eta^{3}-\frac{6848}{21} \log (4 v), \\
\alpha_{7} & =\pi\left(\frac{77096675}{254016}+\frac{378515}{1512} \eta-\frac{74045}{756} \eta^{2}\right) .
\end{aligned}
$$

Among the coefficients above, $\alpha_{5}$ can be simplified further. This interesting possibility arises because of the cancellation of $v^{5}$ of the 2.5PN term with that of the overall factor in the denominator of Eq. (3.3). Consequently, all but the $\ln v$ terms in $\alpha_{5}$ are constants and can be absorbed in a redefinition of the phase ${ }^{3}$. Indeed, we find that all our estimations, except $\Delta \phi_{c}$, remain unchanged irrespective of whether we choose $\alpha_{5}$ as above, or a simplified one retaining only the $\ln v$ term.

In the $3 \mathrm{PN}$ phasing, until recently there were two undetermined parameters, $\lambda$ and $\theta$, arising from the incompleteness of the Hadamard self-field regularisation at $3 \mathrm{PN}^{4}$. By dimensional regularisation $\lambda$ and $\theta$ have been now determined in Ref. 28, 29] and [30, 31, 32, 33] respectively, completing the general relativistic compact inspiral phasing to 3.5PN order: $\lambda=-\frac{1987}{3080} \simeq-0.6451$ and $\theta=-\frac{11831}{9240} \simeq-1.28$. $\lambda$ has also been determined by alternative approach [34].

Following earlier works, we choose the set of independent parameters $\boldsymbol{\theta}$ describing the GW signal to be

$$
\boldsymbol{\theta}=\left(\ln \mathcal{A}, f_{0} t_{c}, \phi_{c}, \ln \mathcal{M}, \ln \eta\right),
$$

where $t_{c}$ refers to the coalescence time, $\phi_{c}$ refers to the phase at coalescence instant, $f_{0}$ is a scaling frequency related to the power spectral density (PSD) of the detectors (see next Subsection). Note that $\mathcal{A}$ is taken to be one of the independent parameters. Computing the Fisher information matrix $\Gamma_{a b}$, whose elements are given by $\left(h_{a} \mid h_{b}\right)($ where $a$ and $b$ are indices which run over the parameters), is the first step towards our goal. The upper cut-off in computing the integrals in Eq. (2.8) and (2.11) is taken to be the GW frequency at the last stable circular orbit (LSO) given, for a test mass in a Schwarzschild spacetime of mass $M$, to be

$$
F_{\text {upper }}=F_{\text {lso }}=\left(6^{3 / 2} \pi M\right)^{-1} .
$$

We take the lower limit in the integrals to be the seismic cut-off frequency $f_{s}$ of the detector.

\section{B. Sensitivity and span of LIGO and VIRGO}

We compute the covariance matrix for three noise curves to understand the effect of detector characteristics on parameter estimation. The noise curves used are advanced LIGO as in 2] and initial LIGO and VIRGO as in 35. We have fitted the following expression to the noise PSD of advanced LIGO given in 2]

$$
\begin{aligned}
S_{h}(f) & =S_{0}\left[x^{-4.14}-5 x^{-2}+\frac{111\left(1-x^{2}+x^{4} / 2\right)}{\left(1+x^{2} / 2\right)}\right], f \geq f_{s} \\
& =\infty, f<f_{s},
\end{aligned}
$$

\footnotetext{
${ }^{3}$ We thank Luc Blanchet for pointing this out to us.

4 The ambiguity parameter $\theta$ occurring at $3 \mathrm{PN}$ should not be confused with the set of parameters $\theta^{a}$ describing the GW.
} 

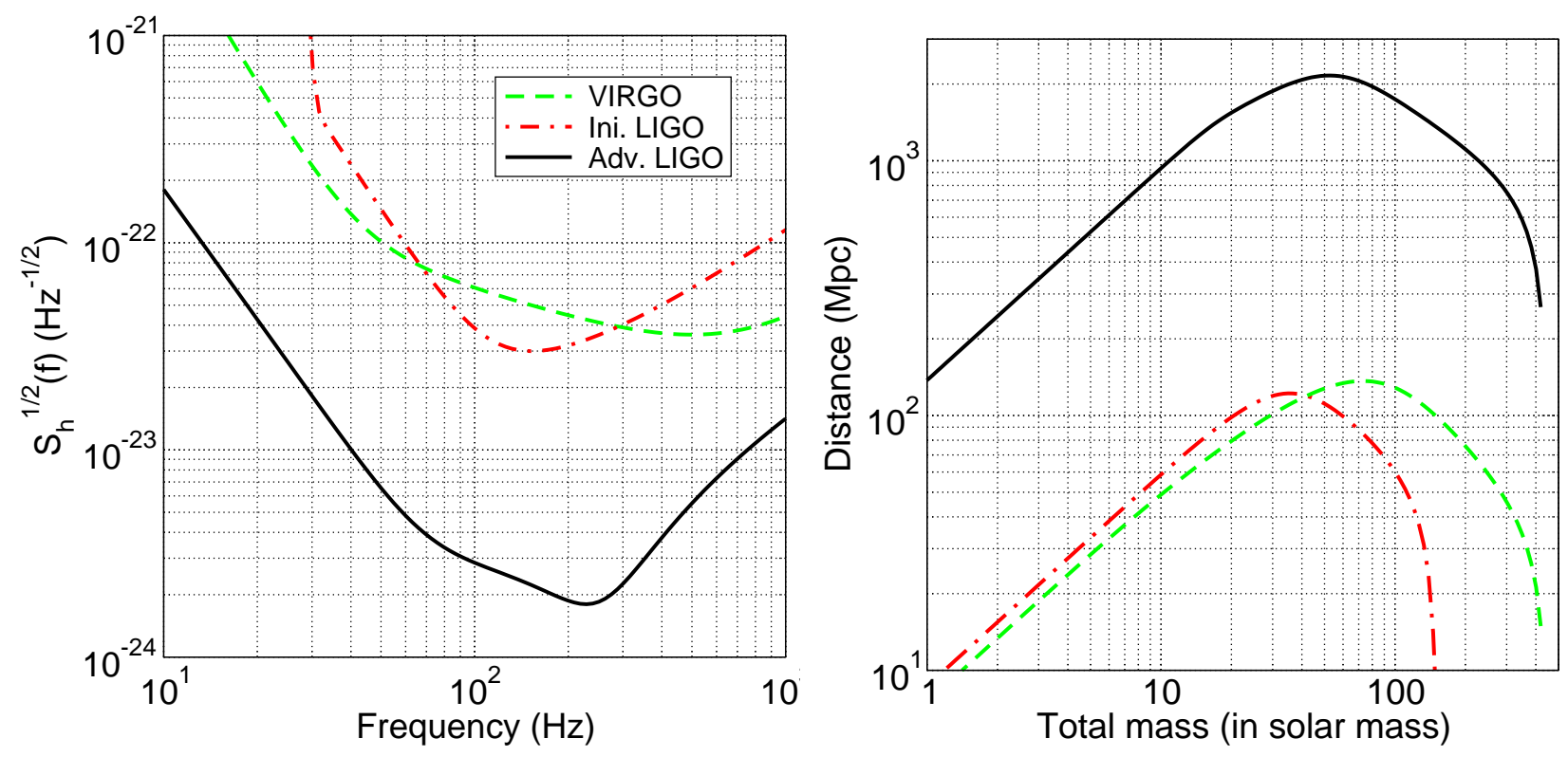

FIG. 1: Amplitude spectrum (left panel) of initial LIGO, VIRGO and advanced LIGO together with the luminosity distance (right panel) at which RMS-oriented binaries would produce a SNR of 5.

where $x=f / f_{0}, f_{0}=215 \mathrm{~Hz}$ (a scaling frequency chosen for convenience), $f_{s}=20 \mathrm{~Hz}$ is the lower cutoff frequency [defined such that for NS-NS binaries the gain in SNR by reducing the lower limit of the integral in Eq. (2.8) below $f_{s}$ is less than $1 \%$ ], and $S_{0}=10^{-49} \mathrm{~Hz}^{-1}$. Note that the above PSD is significantly different from the advanced LIGO noise curve used in earlier studies. Indeed, authors of Ref. [6, 22, 25, 46] use the PSD of advanced LIGO to be $S_{h}(f)=S_{0}\left[x^{-4}+2+2 x^{2}\right], f \geq f_{s}$, and $S_{h}(f)=\infty, f<f_{s}$, with $x=f / f_{0}, f_{0}=70 \mathrm{~Hz}, f_{s}=10 \mathrm{~Hz}$ and $S_{0}=6 \times 10^{-49} \mathrm{~Hz}^{-1}$, which has a significantly better low-frequency sensitivity than what is currently believed to be possible for the next generation of LIGO. Hence, we have chosen to work with the more recent estimate given in Eq. (3.7).

The initial LIGO noise curve from Ref. [35] is given by

$$
\begin{aligned}
S_{h}(f) & =S_{0}\left[(4.49 x)^{-56}+0.16 x^{-4.52}+0.52+0.32 x^{2}\right], f \geq f_{s} \\
& =\infty, f<f_{s},
\end{aligned}
$$

where again $x=f / f_{0}$, with $f_{0}=150 \mathrm{~Hz}, f_{s}=40 \mathrm{~Hz}$ and $S_{0}=9 \times 10^{-46} \mathrm{~Hz}^{-1}$. Finally, for the VIRGO detector the expected PSD is given by [35]:

$$
\begin{aligned}
S_{h}(f) & =S_{0}\left[(6.23 x)^{-5}+2 x^{-1}+1+x^{2}\right], f \geq f_{s} \\
& =\infty, f<f_{s},
\end{aligned}
$$

where $f_{0}=500 \mathrm{~Hz}, f_{s}=20 \mathrm{~Hz}$ and $S_{0}=3.24 \times 10^{-46} \mathrm{~Hz}^{-1}$. The amplitude spectra [i.e. the square-root of the power spectral densities given in Eqs. (3.7)-(3.9)] of the various detectors are plotted in the left hand panel of Fig. [1

The SNR achieved by these detectors for binaries of different masses not only depends on the distance $D$ at which the source is located but also on the orientation of the orbital plane with respect to the line-of-sight. In order not to be biased one can consider binaries of root-mean-square (RMS) orientation and compute the SNR they would produce in a given detector. One can turn around the question and ask the distance at which sources of RMS orientation would produce a specified SNR. Indeed, the distance $D$ at which a binary of RMS orientation achieves a SNR $\rho_{0}$ is given by [37]

$$
D(M, \eta)=\frac{1}{\rho_{0} \pi^{2 / 3}} \sqrt{\frac{2 \eta M^{5 / 3}}{15}}\left[\int_{f_{s}}^{f_{1 \mathrm{so}}(M)} \frac{f^{-7 / 3}}{S_{h}(f)} d f\right]^{1 / 2} .
$$

As is well known the SNR depends only on the chirp mass $\mathcal{M}=\eta^{2 / 3} M$ and not on the masses of the two bodies separately. The SNR is maximum for equal mass binaries (for which $\eta=1 / 4$ ) and is smaller by a factor $\sqrt{4 \eta}$ for systems of the same total mass but consisting of stars of unequal masses. The right-hand panel of Fig. [1 plots the 
TABLE I: Convergence of measurement errors from $1 \mathrm{PN}$ to $3.5 \mathrm{PN}$ at a SNR of 10 for the three prototypical binary systems: NS-NS, NS-BH and BH-BH using the phasing formula, in steps of 0.5PN. For each of the three detector noise curves the table presents $\Delta t_{c}$ (in msec), $\Delta \phi_{c}$ (in radians), $\Delta \mathcal{M} / \mathcal{M}$ and $\Delta \eta / \eta$.

\begin{tabular}{|c|c|c|c|c|c|c|c|c|c|c|c|c|}
\hline \multirow[b]{2}{*}{ PN Order } & \multicolumn{4}{|c|}{ NS-NS } & \multicolumn{4}{|c|}{$\mathrm{NS}-\mathrm{BH}$} & \multicolumn{4}{|c|}{$\mathrm{BH}-\mathrm{BH}$} \\
\hline & $\Delta t_{c}$ & $\Delta \phi_{c}$ & $\Delta \mathcal{M} / \mathcal{M}$ & $\Delta \eta / \eta$ & $\Delta t_{c}$ & $\Delta \phi_{c}$ & $\Delta \mathcal{M} / \mathcal{M}$ & $\Delta \eta / \eta$ & $\Delta t_{c}$ & $\Delta \phi_{c}$ & $\Delta \mathcal{M} / \mathcal{M}$ & $\Delta \eta / \eta$ \\
\hline \multicolumn{13}{|c|}{ Advanced LIGO } \\
\hline $1 \mathrm{PN}$ & 0.3977 & 0.9256 & $0.0267 \%$ & $4.656 \%$ & 0.5959 & 1.261 & $0.1420 \%$ & $7.059 \%$ & 1.162 & 1.974 & $1.041 \%$ & $59.88 \%$ \\
\hline $1.5 \mathrm{PN}$ & 0.4668 & 1.474 & $0.0142 \%$ & $1.638 \%$ & 0.7394 & 2.091 & $0.0763 \%$ & $2.316 \%$ & 1.441 & 3.188 & $0.6115 \%$ & $9.609 \%$ \\
\hline $2 \mathrm{PN}$ & 0.4623 & 1.392 & $0.0143 \%$ & $1.764 \%$ & 0.7208 & 1.848 & $0.0773 \%$ & $2.669 \%$ & 1.404 & 2.850 & $0.6240 \%$ & $10.79 \%$ \\
\hline $2.5 \mathrm{PN}$ & 0.5090 & 1.359 & $0.0134 \%$ & $1.334 \%$ & 0.9000 & 1.219 & $0.0686 \%$ & $1.515 \%$ & 1.819 & 1.574 & $0.5300 \%$ & $5.934 \%$ \\
\hline $3 \mathrm{PN}$ & 0.4938 & 1.331 & $0.0135 \%$ & $1.348 \%$ & 0.8087 & 1.131 & $0.0698 \%$ & $1.571 \%$ & 1.544 & 1.580 & $0.5466 \%$ & $6.347 \%$ \\
\hline $3.5 \mathrm{PN}$ & 0.5193 & 1.279 & $0.0133 \%$ & $1.319 \%$ & 0.9966 & 0.9268 & $0.0679 \%$ & $1.457 \%$ & 2.078 & 1.161 & $0.5241 \%$ & $5.739 \%$ \\
\hline \multicolumn{13}{|c|}{ Initial LIGO } \\
\hline $1 \mathrm{PN}$ & 0.3598 & 1.238 & $0.0771 \%$ & $9.792 \%$ & 0.9550 & 2.510 & $0.5217 \%$ & $20.06 \%$ & 2.406 & 5.038 & $4.750 \%$ & $216.2 \%$ \\
\hline $1.5 \mathrm{PN}$ & 0.4154 & 1.942 & $0.0419 \%$ & $2.768 \%$ & 1.182 & 4.135 & $0.2850 \%$ & $5.410 \%$ & 2.986 & 8.143 & $2.781 \%$ & $28.81 \%$ \\
\hline $2 \mathrm{PN}$ & 0.4109 & 1.816 & $0.0423 \%$ & $3.007 \%$ & 1.148 & 3.597 & $0.2903 \%$ & $6.316 \%$ & 2.900 & 7.179 & $2.851 \%$ & $32.82 \%$ \\
\hline 2.5 & 0.4605 & 1.650 & $0.0384 \%$ & $2.129 \%$ & 1.467 & 1.975 & $0.2491 \%$ & $3.305 \%$ & 3.836 & 3.119 & $2.351 \%$ & $16.48 \%$ \\
\hline $3 \mathrm{PN}$ & 0.4402 & 1.618 & $0.0389 \%$ & $2.170 \%$ & 1.286 & 1.798 & $0.2554 \%$ & $3.474 \%$ & 3.159 & 3.123 & $2.446 \%$ & $17.94 \%$ \\
\hline $3.5 \mathrm{PN}$ & 0.4754 & 1.517 & $0.0383 \%$ & $2.099 \%$ & 1.666 & 1.324 & $0.2456 \%$ & $3.151 \%$ & 4.512 & 1.912 & $2.314 \%$ & $15.77 \%$ \\
\hline \multicolumn{13}{|l|}{ VIRGO } \\
\hline $1 \mathrm{PN}$ & 0.1363 & 0.5134 & $0.0183 \%$ & $3.044 \%$ & 0.4906 & 1.069 & $0.1134 \%$ & $5.782 \%$ & 1.621 & 1.854 & $=0.8745 \%$ & $52.12 \%$ \\
\hline $1.5 \mathrm{PN}$ & 0.1578 & 0.7981 & $0.0098 \%$ & $1.004 \%$ & 0.6069 & 1.763 & $0.0603 \%$ & $1.923 \%$ & 1.430 & 2.972 & $0.5095 \%$ & $8.586 \%$ \\
\hline $2 \mathrm{PN}$ & 0.1562 & 0.7515 & $0.0098 \%$ & $1.085 \%$ & 0.5918 & 1.561 & $0.0611 \%$ & $2.215 \%$ & 1.395 & 2.667 & $0.5199 \%$ & $9.625 \%$ \\
\hline $2.5 \mathrm{PN}$ & 0.1743 & 0.7045 & $0.0091 \%$ & $0.7957 \%$ & 0.7384 & 1.039 & $0.0541 \%$ & $1.263 \%$ & 1.787 & 1.545 & $0.4417 \%$ & $5.370 \%$ \\
\hline $3 \mathrm{PN}$ & 0.1671 & 0.6920 & $0.0092 \%$ & $0.8083 \%$ & 0.6632 & 0.9672 & $0.0551 \%$ & $1.309 \%$ & 1.532 & 1.547 & $0.4552 \%$ & $5.724 \%$ \\
\hline $3.5 \mathrm{PN}$ & 0.1797 & 0.6562 & $0.0091 \%$ & $0.7858 \%$ & 0.8183 & 0.7968 & $0.0536 \%$ & $1.215 \%$ & 2.024 & 1.173 & $0.4369 \%$ & $5.201 \%$ \\
\hline
\end{tabular}

luminosity distance at which binaries of RMS orientation and consisting of stars of equal masses would produce a SNR of $\rho_{0}=5$. After computing the covariance matrix we shall use this plot to study how parameter estimation varies in different interferometers for sources at a fixed distance.

\section{Parameter estimation using 3.5PN phasing - Fixed SNR}

In this Section, we examine how the addition of higher order terms in the phasing formula affects the parameter estimation of the binary. We start from the 1PN phasing formula and add terms in steps of half-a-PN order up to $3.5 \mathrm{PN}$, which is the most accurate expression currently available. We are interested in the case of non-spinning binaries (ignoring spin and orbital angular momentum) and hence estimate only five parameters $\left(\ln \mathcal{A}, f_{0} t_{c}, \phi_{c}, \ln \mathcal{M}, \ln \eta\right)$. We calculate the elements of $\Gamma_{a b}$ by explicitly computing the derivatives of the Fourier domain waveform with respect to (w.r.t) different parameters and taking their noise-weighted inner products. The derivatives and the Fisher matrices are too lengthy to be displayed here. We note that $\Gamma_{1 a}=\delta_{1 a} \rho^{2}$, which renders the Fisher information matrix in block diagonal form. Since $\ln \mathcal{A}$ is now entirely uncorrelated with all other parameters, we only consider the Fisher matrix calculated from the partial derivatives of $\tilde{h}(f)$ with respect to the four parameters $\left(f_{0} t_{c}, \phi_{c}, \ln \mathcal{M}, \ln \eta\right)$. $\Gamma_{11}$ can be thought of as an independent block, and further calculations involving $\mathcal{A}$ become trivial. Finally, by inverting the Fisher information matrix one constructs the covariance matrix.

First, we computed the covariance matrix using the advanced LIGO noise PSD as defined in Ref. 22], which facilitates a comparison of our results with those discussed in the literature. Indeed, at 1.5 PN order we found our results in perfect agreement with the numbers given in Table I of Ref. [22] and at 2PN order our calculation reproduces the results in Table $\mathrm{V}$ of Ref. [26]. In both of these papers, $\ln \mu$, where $\mu$ is the reduced mass, is chosen to be the independent parameter instead of $\ln \eta$. However, the errors in these quantities are simply related by $\frac{\Delta \mu}{\mu}=\frac{2}{5} \frac{\Delta \eta}{\eta}$, so that the comparison is straightforward. In the rest of this paper we study only the most recent advanced LIGO noise PSD together with initial LIGO and VIRGO. 


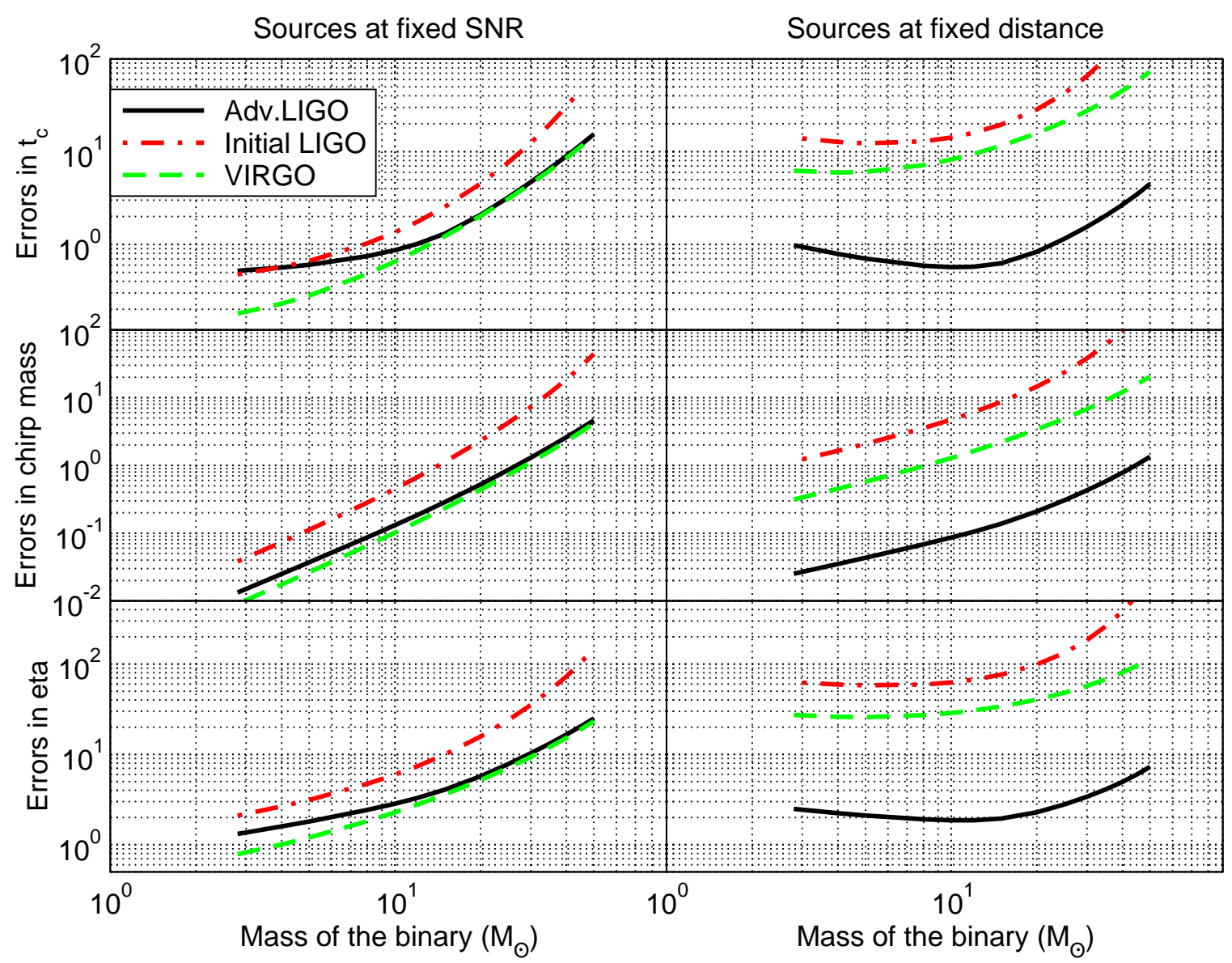

FIG. 2: Comparison of errors in the estimation of $t_{c}, \mathcal{M}$ and $\eta$ for sources with a fixed SNR of 10 (left panels) with those for systems at a fixed distance of $300 \mathrm{Mpc}$ (right panels).

Next, let us consider the covariance matrix computed using the noise PSDs of advanced and initial LIGO, and VIRGO, as given in Eq. (3.7)-(3.9). The errors in the measurement of the various parameters are tabulated in Table [ for all the interferometers and for three prototypical binaries (NS-NS, NS-BH and BH-BH), assuming a fixed SNR of 10 in each case. Although the SNR is fixed, different detectors might accumulate the SNR over different bandwidths, causing the errors to be greater or smaller compared to one another. In agreement with what one expects intuitively based on the bandwidth of the various detectors (cf. Fig. 1, left panel), we find the errors in the various parameters to be the smallest for VIRGO, followed by a factor of roughly 10-70\% larger errors in advanced LIGO compared to VIRGO, and a factor of 3 larger errors in initial LIGO compared to advanced LIGO.

In going from lower to higher post-Newtonian order, we find that there is an 'oscillation' of the errors in the chirp mass and reduced mass. However, the errors at $3.5 \mathrm{PN}$ are always smaller than at $2 \mathrm{PN}$. The opposite oscillation is observed for the errors in $t_{c}$ : the error in $t_{c}$ at $3.5 \mathrm{PN}$ is always higher than at $2 \mathrm{PN}$. The fact that the reduced mass and chirp mass show the same trend is due to the correlation coefficient $c_{\mathcal{M} \eta}$ (listed in Table II) all being close to 1 .

The oscillation in the variances with PN order can be partially understood by an examination of the correlation coefficients between $t_{c}, \mathcal{M}$ and $\eta$. In Table 1 we have listed the correlation coefficients together with the errors in the estimation of parameters in the case of advanced LIGO for a NS-BH system for all PN orders starting from Newtonian but let us first discuss the trend at orders beyond the 1PN correction. From this Table we see that the estimation of $\mathcal{M}$ and $\eta$ improves (degrades) depending on whether the correlation coefficients $c_{\mathcal{M} \eta}$ decrease (respectively, increase) with varying PN order. Similarly, the estimation of $t_{c}$ improves (degrades) depending on whether the correlation coefficients $c_{t_{c} \mathcal{M}}$ (or, equivalently, $c_{t_{c} \eta}$ ) decrease (respectively, increase) with PN order. We have also checked that the estimation of $\phi_{c}$ becomes better (worse) with PN order with reduction (respectively, enhancement) in the correlation coefficients $c_{\phi_{c} \mathcal{M}}$ (or $c_{\phi_{c} \eta}$ ). The same trend is seen for other systems and detector configurations, though we do not list those numbers to avoid proliferation of details. The behaviour of the errors at $0 \mathrm{PN}$ and $1 \mathrm{PN}$ is not in agreement with this general trend because at $0 \mathrm{PN}$ we have only three parameters $-t_{c}, \phi_{c}$ and $\mathcal{M}$. As we go from $1 \mathrm{PN}$ to $1.5 \mathrm{PN}$ the ambiguity function greatly changes its orientation because of the change in sign in the PN series at 1.5PN [cf. 
TABLE II: PN variation in parameter estimation and the associated correlation coefficients for the NS-BH system for the advanced LIGO noise curve.

\begin{tabular}{l|c|c|c|c|c|c}
\hline \hline PN Order & $c_{t_{c} \mathcal{M}}$ & $c_{t_{c} \eta}$ & $c_{\mathcal{M} \eta}$ & $\Delta t_{c}(\mathrm{~ms})$ & $\Delta \mathcal{M} / \mathcal{M}(\%)$ & $\Delta \eta / \eta(\%)$ \\
\hline $0 \mathrm{PN}$ & -0.6451 & - & - & 0.2775 & 0.0255 & - \\
$1 \mathrm{PN}$ & 0.8166 & -0.8810 & -0.9859 & 0.5959 & 0.1420 & 7.059 \\
\hline $1.5 \mathrm{PN}$ & 0.7983 & 0.9280 & 0.9444 & 0.7394 & 0.0763 & 2.316 \\
$2 \mathrm{PN}$ & 0.7947 & 0.9239 & 0.9460 & 0.7208 & 0.0773 & 2.669 \\
$2.5 \mathrm{PN}$ & 0.8145 & 0.9519 & 0.9309 & 0.9000 & 0.0686 & 1.515 \\
$3 \mathrm{PN}$ & 0.8001 & 0.9405 & 0.9333 & 0.8087 & 0.0698 & 1.571 \\
$3.5 \mathrm{PN}$ & 0.8274 & 0.9608 & 0.9294 & 0.9966 & 0.0679 & 1.456 \\
\hline \hline
\end{tabular}

Eq. (3.4c) and Eq. (3.4d)].

Though the PN variation of parameter estimation accuracy seems to be dominantly explained by the variation of the correlation coefficients, it should be borne in mind that the variances in a particular parameter is a combination of the covariances and the availability of a greater structure or variety in the waveforms not fully assessed in this paper. This will be the subject of a study we shall take up in the near future; it is important to understand in more detail why the errors in $t_{c}$ worsen at higher PN orders as it has implications in the determination of the direction to the source. Table III summarizes the results of this Section. It provides the percentage decrease in the errors due to the greater accuracy (3.5PN as opposed to $2 \mathrm{PN}$ ) in the phasing of the waves: the reduction is the highest for a $\mathrm{BH}-\mathrm{BH}$ binary for which the improvement in the estimation of $\eta$ is $52 \%$ and that of $\mathcal{M}$ is $19 \%$ at an SNR of 10 for the initial LIGO noise curve.

\section{Parameter estimation using 3.5PN phasing - Fixed source}

The focus of this Section is to understand the effect of detector sensitivity (as opposed to bandwidth) on parameter estimation. The results of the previous Section, wherein the errors are quoted at a fixed SNR, cannot be used to gauge the performance of different detectors: a more sensitive detector has a larger SNR for a given source and therefore a better estimation of parameters. Hence, we translate the results for the errors in parameter estimation for different detectors but normalized to a fixed distance instead of a fixed SNR. Since the errors associated with the parameter estimation are inversely related to SNR $(\sigma \propto 1 / \rho)$, given the error $\sigma_{0}$ corresponding to a known SNR $\rho_{0}$ (results for $\rho_{0}=10$ are quoted in Table \), one can calculate the error $\sigma$ at another SNR $\rho$ (corresponding to a fixed distance, say, $300 \mathrm{Mpc}$ ) by a simple rescaling of the results listed earlier. Indeed, $\sigma=\rho_{0} \sigma_{0} / \rho$, which can be recast in terms of the distance to the source, using Eq. (3.10), as

$$
\sigma\left(D_{L}\right)=\rho_{0} \sigma_{0} \pi^{2 / 3} D_{L}\left[\frac{2 \eta M^{5 / 3}}{15} \int_{f_{s}}^{f_{1 \mathrm{so}}(M)} \frac{f^{-7 / 3}}{S_{h}(f)} d f\right]^{-1 / 2} .
$$

Fig. 2] summarises the results shown in Table凹(3.5PN entries) over the entire parameter space of interest for sources with a fixed SNR of 10 (left panels) and also the consequent results from the scaling in Eq. (3.11) for sources at a fixed distance of $300 \mathrm{Mpc}$ (right panels). The advantage of having a greater bandwidth is revealed by looking at panels on the left which shows the errors in VIRGO to be the smallest, followed by advanced and initial LIGO instruments. Although the signal-to-noise ratios in the case of VIRGO are similar to those of initial LIGO (cf. Fig. 1 right panel), Fig. 2 reveals that VIRGO measures the parameters more accurately. Indeed, the errors in VIRGO are smaller than in initial LIGO by a factor of 2 to 4 and this is entirely as a result of VIRGO's larger bandwidth. Unlike the case of fixed SNR, detector performance is explicit in the plots for sources at a fixed distance. It is evident that the errors reduce by about 30-60 times in advanced LIGO as compared to initial LIGO. Advanced LIGO gains a factor of 10-15 in SNR relative to initial LIGO and this accounts for most of the improvement in its parameter estimation. However, it also gains another factor of 3 to 4 because of its greater bandwidth. From the foregoing discussion we conclude that as far as parameter estimation is concerned VIRGO performs better than initial LIGO and that advanced LIGO can measure the parameters significantly better than what one might conclude based on the improvement over VIRGO in its visibility of the signals.

A final comment: The plots on the right-hand panel of Fig. 2 are somewhat flattened as compared to those on the left-hand panel due to the fact that errors for sources at a fixed distance are (anti) correlated with the variation of SNR with mass. In other words, there are two competing effects on parameter estimation as the mass of the 
TABLE III: Percentage change of parameter estimation accuracy at SNR $\rho=10$ for non-spinning compact binaries due to improved phasing accuracy from $2 \mathrm{PN}$ to $3.5 \mathrm{PN}$. Percentage change for the parameter $\sigma_{n}$ is taken to be $=100 \times\left(1-\sigma_{n}^{3.5 \mathrm{PN}} / \sigma_{n}^{2 \mathrm{PN}}\right)$. Negative values imply worsened parameter estimation in going from $2 \mathrm{PN}$ to $3.5 \mathrm{PN}$.

\begin{tabular}{|c|c|c|c|c|c|c|c|c|c|c|c|}
\hline \multirow[b]{2}{*}{ Interferometer } & \multicolumn{3}{|c|}{ NS-NS } & \multicolumn{4}{|c|}{ NS-BH } & \multicolumn{4}{|c|}{$\mathrm{BH}-\mathrm{BH}$} \\
\hline & $t_{c}$ & $\phi_{c}$ & $\ln \mathcal{M} \ln \eta$ & $t_{c}$ & $\phi_{c}$ & $\ln \mathcal{M}$ & $\ln \eta$ & $t_{c}$ & $\phi_{c}$ & $\ln \mathcal{M}$ & $\ln \eta$ \\
\hline Adv. LIGO & -12.33 & 8.118 & 6.99325 .23 & -38.26 & 49.85 & 12.16 & 45.41 & -48.01 & 59.26 & 16.01 & 46.81 \\
\hline Ini. LIGO & -15.70 & 16.47 & 9.45630 .20 & -45.12 & 63.19 & 15.40 & 50.11 & -55.59 & 73.37 & 18.84 & 51.95 \\
\hline VIRGO & -15.05 & 12.68 & $7.143 \quad 27.58$ & -38.27 & 48.96 & 12.28 & 45.15 & -45.09 & 56.02 & 15.97 & 45.96 \\
\hline
\end{tabular}

binary is increased. On the one hand, estimation becomes worse since the signal spends smaller amount of time in the detector band and the number of cycles available to discriminate different signals goes down. On the other hand, as we increase the mass of the binary the SNR increases thereby aiding in discriminating between different systems. These competing trends cause the error in the estimation of the time-of-coalescence and symmetric mass ratio to show a minimum for a binary of total mass $M \sim 10 M_{\odot}$. No such minimum is seen, however, in the case of the chirp mass. This is because the error in the chirp mass rises more steeply with mass than the SNR can cause it to dip.

\section{E. Parameter estimation and Number of useful cycles}

To investigate further the correlation of parameter estimation performance with detector characteristics we consider the total number of cycles in the detector bandwidth and more importantly the number of useful cycles for a particular detector for the three systems under consideration. The total number of cycles $N_{\text {total }}$, is defined as

$$
N_{\text {total }}=\int_{F_{\text {begin }}}^{F_{\text {end }}} d F\left(\frac{1}{2 \pi} \frac{d \phi}{d F}\right)
$$

where $\phi$ is the phase of the GW, $F_{\text {begin }}$ and $F_{\text {end }}$ correspond to the upper and lower cut-off frequencies for the astrophysical system under consideration. Since the phasing of the waves is a post-Newtonian expansion in the parameter $v$ the total number of cycles depends on the post-Newtonian order. At the dominant Newtonian order, assuming that the lower frequency cutoff of the detector is much smaller compared to the last stable orbit frequency of the system, the total number of cycles for a binary of total mass $M$ and mass ratio $\eta$ is given by

$$
N_{\text {total }}=\frac{\left(\pi M f_{s}\right)^{-5 / 3}}{32 \pi \eta}
$$

The total number of cycles goes inversely as the mass ratio being the smallest (for a given total mass) for equal mass binaries and is quite a sharp function of the total mass. It has an artificiality to it in that it depends on the chosen lower-frequency cutoff, increasing quite rapidly as the the cutoff is lowered. Moreover, $N_{\text {total }}$ has no information about detector characteristics. Motivated by these facts Ref. [37] proposed that the detector performance can be better understood using the idea of the number of useful cycles $N_{\text {useful }}$ defined as

$$
N_{\text {useful }}=\left[\int_{F_{\min }}^{F_{\max }} \frac{d f}{f} w(f) N(f)\right]\left[\int_{F_{\min }}^{F_{\max }} \frac{d f}{f} w(f)\right]^{-1}
$$

where $N(F)$ is the instantaneous number of cycles (i.e., the number of cycles spent at the instantaneous frequency $F$ ) and $w(f)$ is the weighting function that depends on the effective noise of the interferometer and the amplitude of the source defined as

$$
N(F)=\frac{F^{2}}{d F / d t}, \quad w(f)=\frac{a^{2}(f)}{h_{n}^{2}(f)}
$$

with $a(f)$ being the 'bare amplitude' appearing in the Fourier domain waveform within the SPA, $|\tilde{h}(f)| \simeq a(f) / \sqrt{\dot{F}}$ and $h_{n}^{2} \equiv f S_{h}(f)$. Unlike the total number of cycles, the number of useful cycles contains information about both the detector and the source: it is weighted by the noise PSD of the instrument and amplitude of the source. Moreover, while the total number of cycles depends critically on the choice of the lower-cutoff, the number of useful cycles is a 

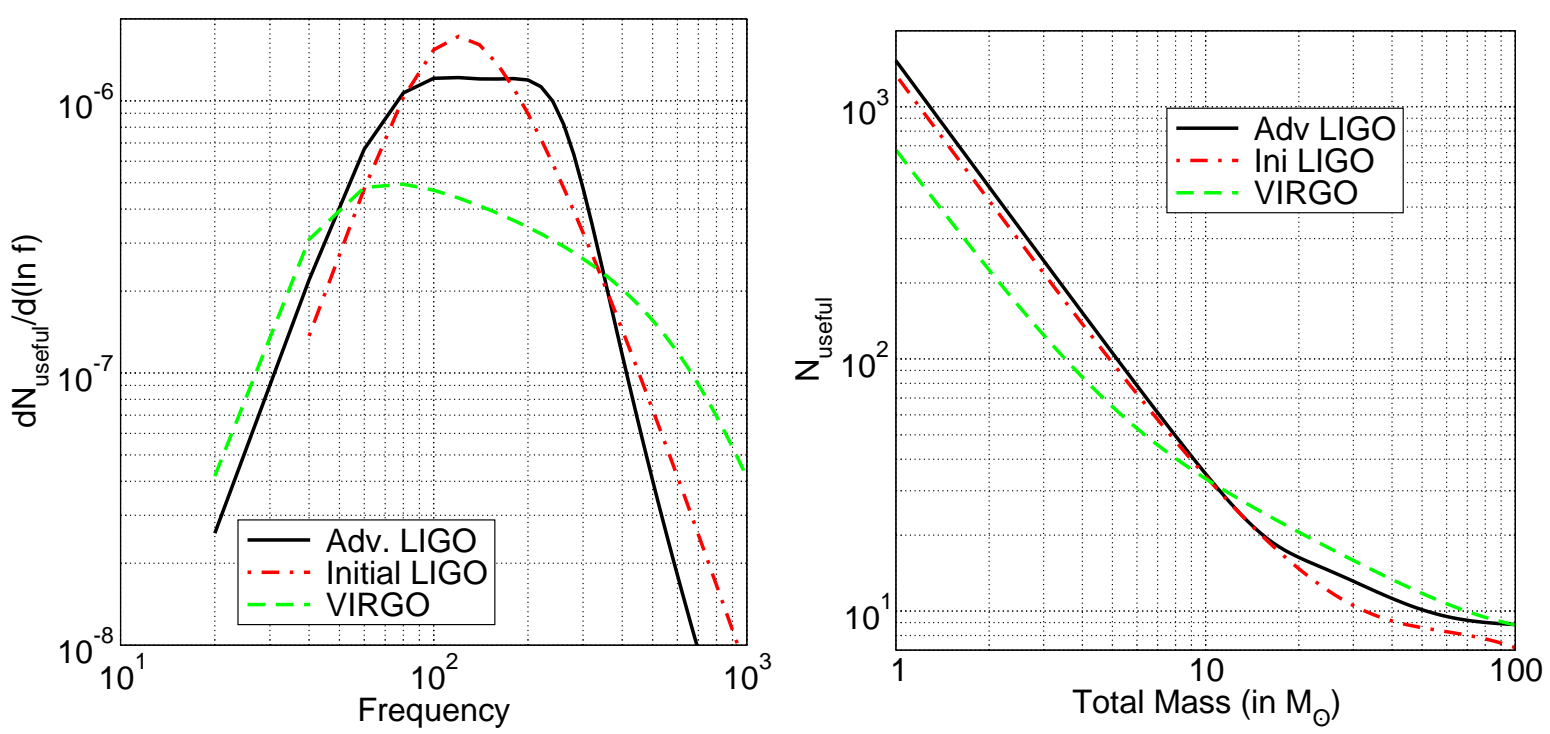

FIG. 3: Left hand panel is the plot of the derivative $d N_{\text {useful }} / d(\ln f)$ against the frequency (in arbitrary normalization) for the three detectors. Similarly, right panel gives the number of useful cycles as a function of the total mass of the binary for the three detectors.

robust estimator and it is pretty much independent of the cutoffs chosen as long as the frequency range covers the sensitivity bandwidth of the instrument.

At Newtonian order, the instantaneous number of cycles is given by $N(f)=5(\pi M f)^{-5 / 3} /(96 \eta)$, which clearly exhibits the well-known fact that irrespective of the mass of the system it is best to design a detector with a good sensitivity at as low a frequency as possible. The instantaneous number of cycles decreases rapidly with frequency, but most of the contribution to the integral in Eq. (3.14) comes from the region of the band where weighting function $w(f)=a^{2}(f) / h_{s}^{2}(f)=f^{1 / 3} / S_{h}(f)$, has a minimum. As shown in Fig. [3 (right-hand panel) for binaries whose total mass is larger than $11 M_{\odot}$ the number of useful cycles is larger in VIRGO than the other two instruments, while just the opposite is true for systems whose mass is smaller than $11 M_{\odot}$. The reason for this behaviour can be seen by inspecting the left-hand panel of Fig. 3 where we have plotted the integrand $d N_{\text {useful }} / d \log f$ of the number of useful cycles [cf. Eq. (3.14)]. A binary of total mass $100 M_{\odot}$ has its last stable orbit at $F_{\text {lso }} \simeq 43\left(M / 100 M_{\odot}\right)^{-1} \mathrm{~Hz}$, and increases in inverse proportion to the mass for systems with lower masses. Since the integral in Eq. (3.14) is terminated at $F_{\text {lso }}$, from Fig. 3 we see that as the upper limit of the integral increases (equivalently, the mass of the binary decreases) at first the number of useful cycles for VIRGO begins to increase. This feature explains why VIRGO has more number of cycles than the LIGO instruments for binaries with greater masses. However, owing to their relatively narrower bandwidth (as compared to VIRGO) both the LIGO instruments quickly catch up and for $F_{\text {lso }} \gtrsim 300 \mathrm{H} z$, (equivalently, a total mass of $M \lesssim 14 M_{\odot}$ ), they have greater number of useful cycles than VIRGO. Thus, the relatively broader bandwidth of VIRGO is responsible for the smaller number of useful cycles at lower masses.

In general, one can correlate the larger errors associated with the estimation of parameters of massive systems with the smaller number of useful cycles for these systems (see Table IV]. It may be recalled that Ref. [37] showed that the number of useful cycles is a good quantifier of detector performance with regard to detection issues such as effectualness. However, the efficiency in parameter estimation is a combination of bandwidth and the number of useful cycles and not the latter alone. Thus, though VIRGO has a smaller number of useful cycles than the two LIGO detectors for the NS-NS system, its parameter estimation at a fixed SNR is far better because of its broader bandwidth.

Following Ref. [25], where the effects induced in parameter estimation due to the inclusion of the $2 \mathrm{PN}$ term was understood in terms of the additional total number of GW cycles accumulated at that order, we also use a very similar idea to understand the PN variations in parameter estimation of Table \ But unlike 25], we use the number of useful cycles instead of the total number of cycles. From Table $\nabla$ wherein we have given the errors in chirp mass and symmetric mass ratio together with the contributions to the useful GW cycles from each PN order term in phasing, it is obvious that, in general, when the number of cycles increases in going from one order to another, errors decrease (and vice versa) suggesting a possible correlation. Further, following [25], we tested this argument by artificially flipping the sign of each PN order term in the phasing (keeping all lower order terms with the correct sign) and comparing the errors. If such a correlation exists, one would expect the trend to be reversed, as the additional 
TABLE IV: Number of useful cycles (and total number of cycles in brackets) for different systems and different detectors computed using 3.5PN phasing. To compute the total number of cycles the lower cut-off is chosen to be the seismic cutoff frequency of each detector and the upper cutoff is the frequency corresponding to the LSO.

\begin{tabular}{c|c|c|c}
\hline \hline Detector & NS-NS & NS-BH & BH-BH \\
\hline Adv. LIGO & $284(5136)$ & $60(1111)$ & $14(184)$ \\
Ini. LIGO & $251(1615)$ & $59(330)$ & $12(52)$ \\
VIRGO & $140(5136)$ & $64(1111)$ & $18(184)$ \\
\hline \hline
\end{tabular}

number of useful cycles accumulated reverses its sign. Indeed Case B of the table does show the opposite trend confirming this correlation. There is an important exception to this correlation while going from Newtonian to 1PN, where though the number of useful cycles increase, the parameter estimation worsens. A little thought reveals that another more dominant aspect comes into play at this order due to the inclusion of the new parameter $\eta$ which could increase the errors associated with the original set of parameters. This is confirmed by looking at the parameter estimation of the Newtonian and $1 \mathrm{PN}$ orders using a smaller set of four parameters i.e. $\left\{\ln \mathcal{A}, t_{c}, \phi_{c}, \ln \mathcal{M}\right\}$, excluding $\ln \eta$. We find that the percentage error in chirp mass decreases from 0.0126 to 0.0120 for NS-NS case and 0.4833 to 0.4183 in the $\mathrm{BH}-\mathrm{BH}$ case in step with the increase in number of useful cycles. However, the reason behind the anomalous behaviour in going from 1 to $1.5 \mathrm{PN}$ and 3 to $3.5 \mathrm{PN}$ - where despite the decrease in the number of useful cycles, the parameter estimation improves - is not clear from the present analysis. Thus the previous considerations are not sufficient to completely understand the variation of parameter estimation with the PN order.

Based on the understanding obtained in the previous paragraph, we conclude the Section with the following comment: At present we do not have a detailed understanding of the reason underlying the variation in parameter estimation with PN orders since the inclusion of higher PN terms could lead to one or more of the following: (a) introduction of a new parameter (e.g. $\eta$ in going from $0 \mathrm{PN}$ to $1 \mathrm{PN}$ ) leading to an increase in the variance of the existing parameters, (b) increase in the 'variety' of waveforms leading to a reduction in the variance, and (c) change in the covariance among the various parameters. Though by a critical examination of the results summarised in Tables 【 and [1] some of these effects can be seen in action, it is not easy to disentangle these individual effects and present a consistent quantitative picture. This, we leave to a future study.

\section{BEYOND THE RESTRICTED WAVEFORM: AMPLITUDE CORRECTIONS DUE TO FREQUENCY-SWEEP AND ITS IMPLICATIONS}

In the foregoing Sections we worked with the restricted PN approximation. In this approximation the GW phase is taken to as high a PN accuracy as available while the amplitude is assumed to be Newtonian. Indeed, all harmonics, except the dominant one at twice the orbital frequency, are neglected. From Eq. (3.1), one can see that the Fourier-domain amplitude is determined by the product of the time-domain amplitude $A(F) \propto F^{2 / 3}$ and the factor $(d F / d t)^{-1 / 2}$, where $d F / d t$ is the 'frequency-sweep' or 'chirp rate' of the signal. The frequency-sweep provides a way of (partially) computing the dependence of the wave amplitude on different PN orders. This correction, in addition to being calculable, should be of some relevance when we compared in Sec. IIB parameter estimation accuracy at different PN orders where, following Ref. [25], we assumed the SNR to be the same at all PN orders. Our assumption was justified since in the restricted PN approximation there is no change in the amplitude of the signal as we go from one PN order to the next. However, the frequency-sweep causes the Fourier amplitude to change across the PN orders and leads to variations in the SNR with the PN orders. Since the errors depend on the SNR, one should rescale the errors by the ratio of SNRs to compare fairly the PN trends in parameter estimation of the chirp signal. In what follows, we will set up the necessary formulas to normalize the errors to the same SNR. However, it is immediately obvious that a more consistent calculation should begin with the full amplitude corrections arising from the GW polarizations computed in Ref. 11, 12], in lieu of the restricted approximation used here, and by including the sub-dominant harmonics. Inclusion of these terms is beyond the scope of this paper and will be addressed elsewhere.

To estimate the amplitude corrections due to the frequency-sweep $\dot{F}$, we start from the Fourier domain waveform in the stationary phase approximation which can be written as

$$
\tilde{h}(f) \equiv \int_{-\infty}^{\infty} h(t) e^{-2 \pi i f t} d t=\left[2 \eta \frac{M}{d} Q(\text { angles })\right] \frac{v^{2}}{\sqrt{\dot{F}(v)}} e^{i \psi(f)},
$$

where $v=(\pi M f)^{1 / 3}$. Using the expression for $\dot{F}$ at the Newtonian order, it can easily be shown that Eq. (4.1) reduces to Eq. (3.2). From Eq. (4.1) it is clear that the PN corrections in the frequency-sweep $\dot{F}$ [see Eq. (4.8) below] 
TABLE V: Correlation of parameter estimation and number of useful cycles with PN order $(n)$ for NS-NS and BH-BH Binaries for initial LIGO noise curve. Case A corresponds to the standard PN coefficients in the phasing formula $\left(\epsilon_{a}=1, a \leq n\right)$. Case $\mathrm{B}$ refers to the results corresponding to a flip in sign of the $a=n P N$ term keeping all other lower orders with correct sign $\left(\epsilon_{a}=-1\right.$ for $a=n$ and 1 for $\left.a<n\right)$. Errors listed are all in percentages. The values for the Newtonian order are obtained using a set of four parameters, $\left\{\ln \mathcal{A}, t_{c}, \phi_{c}, \ln \mathcal{M}\right\}$, excluding $\ln \eta$.

\begin{tabular}{|c|c|c|c|c|c|c|}
\hline \multirow[b]{2}{*}{ PN Order $(n)$} & \multicolumn{3}{|c|}{ NS-NS } & \multicolumn{3}{|c|}{$\mathrm{BH}-\mathrm{BH}$} \\
\hline & $\Delta \mathcal{M} / \mathcal{M}$ & $\Delta \eta / \eta$ & $N_{\text {useful }}$ & $\Delta \mathcal{M} / \mathcal{M}$ & $\Delta \eta / \eta$ & $N_{\text {useful }}$ \\
\hline \multicolumn{7}{|l|}{ Case A } \\
\hline $0 \mathrm{PN}$ & 0.0126 & & 247.8 & 0.4833 & & 14.98 \\
\hline $1 \mathrm{PN}$ & 0.0771 & 9.792 & 27.13 & 4.750 & 216.2 & 7.283 \\
\hline $1.5 \mathrm{PN}$ & 0.0419 & 2.768 & -22.98 & 2.781 & 28.81 & -9.148 \\
\hline $2 \mathrm{PN}$ & 0.0423 & 3.007 & -1.197 & 2.851 & 32.82 & -0.496 \\
\hline $2.5 \mathrm{PN}$ & 0.0384 & 2.129 & 2.406 & 2.351 & 16.48 & 1.850 \\
\hline $3 \mathrm{PN}$ & 0.0389 & 2.170 & -1.735 & 2.446 & 17.94 & -1.971 \\
\hline $3.5 \mathrm{PN}$ & 0.0383 & 2.098 & -0.151 & 2.313 & 15.75 & -0.236 \\
\hline \multicolumn{7}{|l|}{ Case B } \\
\hline $1 \mathrm{PN}$ & 0.0771 & 8.858 & -21.65 & 4.750 & 158.2 & -3.579 \\
\hline $1.5 \mathrm{PN}$ & 0.0547 & 1.842 & 80.65 & 3.237 & 28.04 & 21.56 \\
\hline $2 \mathrm{PN}$ & 0.0415 & 2.564 & -53.64 & 2.727 & 25.63 & -19.30 \\
\hline $2.5 \mathrm{PN}$ & 0.0515 & 5.085 & -4.700 & 14.96 & 473.7 & -2.395 \\
\hline $3 \mathrm{PN}$ & 0.0380 & 2.089 & 6.563 & 2.271 & 15.26 & 6.461 \\
\hline $3.5 \mathrm{PN}$ & 0.0395 & 2.248 & -3.453 & 2.625 & 20.89 & -4.978 \\
\hline
\end{tabular}

introduces a related PN correction in the amplitude as discussed earlier in the Section. To proceed further we note that the formula for $\dot{F}$ can be normalized w.r.t. its Newtonian value $\dot{F}_{\mathcal{N}}$ and written as the product of the Newtonian value and PN corrections $\dot{F}_{\mathcal{C}}$ :

$$
\dot{F}=\dot{F}_{\mathcal{N}} \dot{F}_{\mathcal{C}}
$$

Schematically $\dot{F}_{\mathcal{C}}$ can be written as

$$
\dot{F}_{\mathcal{C}}=\left[1+\dot{F}_{\mathcal{C}}^{1 P N}+\dot{F}_{\mathcal{C}}^{1.5 P N}+\dot{F}_{\mathcal{C}}^{2 P N}+\dot{F}_{\mathcal{C}}^{2.5 P N}+\dot{F}_{\mathcal{C}}^{3 P N}+\dot{F}_{\mathcal{C}}^{3.5 P N}+\cdots\right] .
$$

Using $F_{\mathcal{N}}=\frac{96}{5 \pi \mathcal{M}^{2}}(\pi \mathcal{M} F)^{11 / 3}$ and $v=(\pi M F)^{1 / 3}$ and some simple algebra, one can write,

$$
\tilde{h}_{\mathcal{C}}(f)=\mathcal{B}_{\mathcal{N}} \mathcal{B}_{\mathcal{C}} e^{i \psi(f)}, \quad \mathcal{B}_{\mathcal{N}}=\mathcal{A} f^{-7 / 6}, \quad \mathcal{B}_{\mathcal{C}}=\frac{1}{\sqrt{\dot{F}_{\mathcal{C}}}}
$$

where $\mathcal{B}_{\mathcal{N}}$, as in Eq. (2.8), is the Newtonian functional dependence. Using Eq. (4.4), the expression for SNR can be re-written as

$$
\rho^{2}=4 \int_{0}^{\infty} d f \mathcal{B}_{\mathcal{C}}^{2} \frac{\mathcal{B}_{\mathcal{N}}^{2}}{S_{h}(f)}
$$

From the definition of SNR, Eq. (2.8), it is clear that the SNR varies with the PN order of $\dot{F}_{\mathcal{C}}$. Similarly, one can write down the components of the Fisher matrix $\Gamma_{a b}$ as

$$
\Gamma_{a b}=2 \int_{0}^{+\infty} d f \frac{\mathcal{B}_{\mathcal{N}}^{2}}{S_{h}(f)}\left[\frac{\partial \mathcal{B}_{\mathcal{C}}}{\partial \theta_{a}} \frac{\partial \mathcal{B}_{\mathcal{C}}}{\partial \theta_{b}}+\mathcal{B}_{\mathcal{C}}{ }^{2} \frac{\partial \psi}{\partial \theta_{a}} \frac{\partial \psi}{\partial \theta_{b}}\right]
$$

where $\theta_{a}$ and $\theta_{b}$ are the parameters in the GW signal. $\left(\mathcal{B}_{\mathcal{C}}\right.$ is a PN series in $v$ and its $\theta$ dependence arises solely from the mass dependence of $v$ ). In Sec III $\mathcal{B}_{\mathcal{C}}$ was effectively taken to be unity. Here we relax that assumption by taking into account the PN corrections involved. 
TABLE VI: Variation in SNR with the PN order due to the amplitude corrections arising from the frequency-sweep $d F / d t$ in the stationary phase approximation. We assume initial LIGO noise spectral density and place the source such that at $0 \mathrm{PN}$ order we have a SNR of 10 .

\begin{tabular}{l|r|r|r}
\hline \hline PN Order & NS-NS & NS-BH & BH-BH \\
\hline $0 \mathrm{PN}$ & 10.00 & 10.00 & 10.00 \\
$1 \mathrm{PN}$ & 10.53 & 11.26 & 12.19 \\
$1.5 \mathrm{PN}$ & 10.08 & 9.560 & 9.357 \\
$2 \mathrm{PN}$ & 10.06 & 9.483 & 9.178 \\
$2.5 \mathrm{PN}$ & 10.11 & 9.736 & 9.861 \\
$3 \mathrm{PN}$ & 10.08 & 9.355 & 9.157 \\
$3.5 \mathrm{PN}$ & 10.07 & 9.341 & 9.060 \\
\hline \hline
\end{tabular}

The frequency-sweep appearing in Eq. (4.1) above can be straightforwardly calculated from the expressions for the flux function $\mathcal{F}$ and the energy function $E$, determining the GW phasing in the adiabatic approximation. It is given by 35

$$
\dot{F}(v)=-\frac{3 v^{2}}{\pi M^{2}} \frac{\mathcal{F}(v)}{E^{\prime}(v)},
$$

where $v=(\pi M f)^{1 / 3}$ and $E^{\prime}=\frac{d E}{d v}$. Using the 3.5PN accurate expression for $E$ and $\mathcal{F}$ available in [10], the expression for $\dot{F}$ up to $3.5 \mathrm{PN}$ is given by

$$
\begin{aligned}
\left(\frac{d F}{d t}\right)^{3.5 P N}= & \frac{96}{5 \pi \mathcal{M}^{2}}(\pi \mathcal{M} F)^{11 / 3}\left[1-\left(\frac{743}{336}+\frac{11}{4} \eta\right)(\pi M F)^{2 / 3}+(4 \pi)(\pi M F)\right. \\
& +\left(\frac{34103}{18144}+\frac{13661}{2016} \eta+\frac{59}{18} \eta^{2}\right)(\pi M F)^{4 / 3}+\left(-\frac{4159 \pi}{672}-\frac{189 \pi}{8} \eta\right)(\pi M F)^{5 / 3} \\
& +\left[\frac{16447322263}{139708800}+\frac{16 \pi^{2}}{3}-\frac{1712}{105} \gamma+\left(-\frac{273811877}{1088640}+\frac{451 \pi^{2}}{48}-\frac{88}{3} \theta+\frac{616}{9} \lambda\right) \eta\right. \\
& \left.+\frac{541}{896} \eta^{2}-\frac{5605}{2592} \eta^{3}-\frac{856}{105} \log (16 x)\right](\pi M F)^{2} \\
& \left.+\left(-\frac{4415}{4032}+\frac{358675}{6048} \eta+\frac{91495}{1512} \eta^{2}\right) \pi(\pi M F)^{7 / 3}\right]
\end{aligned}
$$

In the above expression $\gamma$ is the Euler's constant $(\gamma=0.577 \cdots)$ and the coefficients $\lambda=-\frac{1987}{3080} \simeq-0.6451, \theta=$ $-\frac{11831}{9240} \simeq-1.28$.

In Table VI] we summarize how the SNR varies with the PN order for different sources assuming that the SNR corresponding to the Newtonian order is 10. The convergence of the SNR's with PN orders is pretty obvious, although it should be recalled that the complete waveform includes PN corrections from other harmonics that are comparable to the higher order terms in the frequency-sweep [11, 12]. It would be interesting to see how the results change when these are included. We also note that the variation of the SNR is greater for systems with larger masses. Using a 3.5PN frequency-sweep, instead of the Newtonian one, increases the SNR by $0.7 \%$ for a NS-NS binary, while the SNR decreases by $9.5 \%$ for a BH-BH binary. Though these amplitude corrections may not be important for NS-NS binaries, they might be relevant for the $\mathrm{BH}-\mathrm{BH}$ case.

Using the results in Table VI one can implement a simple procedure to obtain better error estimates. One can scale the results of Sec. III obtained within the restricted waveform approximation, by the factor $\rho_{n} / \rho_{0}$, where $\rho_{n}$ and $\rho_{0}$ are the SNRs at $n \mathrm{PN}$ and $0 \mathrm{PN}$ orders, respectively. In this simple estimate one is effectively neglecting the contributions to the Fisher matrix from the variation of the $\dot{F}$ terms in the amplitude w.r.t the signal parameters $\boldsymbol{\theta}$ (see Eq. (4.6). We incorporate this contribution in a more general and rigorous way in what follows.

Our more general procedure is based on Eqs. (4.5) and (4.6) which accounts for the SNR and the Fisher matrix, respectively, with the full $\dot{F}$ dependence in amplitude. The steps leading to the final results listed in Table VII are as follows: (i) compute the amplitude $\mathcal{A}$ such that the SNR at $0 \mathrm{PN}$ is 10; (ii) compute the Fisher matrix taking into account the amplitude corrections from the frequency-sweep using Eq. (4.6); (iii) scale the final results by $\rho_{n} / \rho_{0}$. 
TABLE VII: Parameter estimation with amplitude corrections from the frequency-sweep incorporated for the initial LIGO noise curve and SNR $\rho=10$. $n \mathrm{PN}$ refers to the choice of $n^{t h} \mathrm{PN}$ order both in the amplitude and phase of the frequency-domain waveform

\begin{tabular}{|c|c|c|c|c|c|c|c|c|c|c|c|c|}
\hline \multirow[b]{2}{*}{ PN order } & \multicolumn{4}{|c|}{ NS-NS } & \multicolumn{4}{|c|}{ NS-BH } & \multicolumn{4}{|c|}{ BH-BH } \\
\hline & $\Delta t_{c}$ & $\Delta \phi_{c}$ & $\Delta \mathcal{M} / \mathcal{M}$ & $\Delta \eta / \eta$ & $\Delta t_{c}$ & $\Delta \phi_{c}$ & $\Delta \mathcal{M} / \mathcal{M}$ & $\Delta \eta / \eta$ & $\Delta t_{c}$ & $\Delta \phi_{c}$ & $\Delta \mathcal{M} / \mathcal{M}$ & $\Delta \eta / \eta$ \\
\hline $1 \mathrm{PN}$ & 0.3208 & 1.157 & $0.0752 \%$ & $9.431 \%$ & 0.9077 & 2.449 & $0.5241 \%$ & $19.94 \%$ & 1.717 & 3.544 & $3.400 \%$ & $152.6 \%$ \\
\hline $1.5 \mathrm{PN}$ & 0.4417 & 2.018 & $0.0426 \%$ & $2.851 \%$ & 1.234 & 4.232 & $0.2840 \%$ & $5.484 \%$ & 2.929 & 7.883 & $2.647 \%$ & $27.71 \%$ \\
\hline $2 \mathrm{PN}$ & 0.4427 & 1.903 & $0.0432 \%$ & $3.115 \%$ & 1.206 & 3.695 & $0.2892 \%$ & $6.408 \%$ & 2.830 & 6.897 & $2.680 \%$ & $31.24 \%$ \\
\hline $2.5 \mathrm{PN}$ & 0.4693 & 1.672 & $0.0387 \%$ & $2.149 \%$ & 1.496 & 2.009 & $0.2480 \%$ & $3.326 \%$ & 3.801 & 3.093 & $2.316 \%$ & $16.28 \%$ \\
\hline $3 \mathrm{PN}$ & 0.4768 & 1.709 & $0.0398 \%$ & $2.261 \%$ & 1.387 & 1.919 & $0.2556 \%$ & $3.583 \%$ & 3.106 & 3.068 & $2.307 \%$ & $17.20 \%$ \\
\hline $3.5 \mathrm{PN}$ & 0.5186 & 1.615 & $0.0393 \%$ & $2.199 \%$ & 1.787 & 1.435 & $0.2459 \%$ & $3.260 \%$ & 4.474 & 1.939 & $2.203 \%$ & $15.33 \%$ \\
\hline
\end{tabular}

The covariance matrix obtained from such a procedure can then be compared with that obtained in Sec. IIII The procedure above is obviously equivalent to choosing a 'running' amplitude $\mathcal{A}_{n}$, with $\rho_{0}=10$.

In Table VII the variation of errors with different PN orders is shown for the initial LIGO noise curve ${ }^{5}$. The oscillation of errors with PN orders remains after the inclusion of the frequency-sweep and one infers that changes due to these amplitude terms are not very significant. At an SNR of 10 the difference is at most $10 \%$.

\section{CONCLUSION}

\section{A. Summary and discussion of results}

We have carried out a detailed study to understand the implication of 3.5PN phasing formula on parameter estimation of non-spinning binaries using the covariance matrix. We also compare parameter estimation using three different noise curves, advanced LIGO, initial LIGO, and VIRGO. The results of our study can be summarised as follows:

1. The parameter estimation of non-spinning binaries improves significantly, as expected, by employing the 3.5PN phasing formula instead of the $2 \mathrm{PN}$ one. It is no surprise that the same trend is observed for all the three detectors. Improvements are larger for NS-BH and BH-BH systems and least for NS-NS binary. For initial LIGO, at a SNR of 10 , the improvement in the estimation of parameters $\mathcal{M}$ and $\eta$ for $\mathrm{BH}-\mathrm{BH}$ binaries is as large as $19 \%$ and $52 \%$, respectively, whereas for NS-BH binaries it is $15 \%$ and $50 \%$. Improvements in the case of VIRGO are slightly less compared to LIGO (cf. Table III).

2. In proceeding from $1 \mathrm{PN}$ to $3.5 \mathrm{PN}$, one sees an oscillation of variances with each half $\mathrm{PN}$ order. However, the errors in the mass parameters at $3.5 \mathrm{PN}$ are always smaller than at $1 \mathrm{PN}$ and one can see a convergence within this limited sequence. The oscillation of errors is a characteristic feature of the PN approximation. In Ref. 47], a similar oscillatory behaviour is seen in the context of the detection problem. The variation in parameter estimation accuracies with PN orders seem to be dominantly determined by the covariances between the parameters $t_{c}, \phi_{c}, \mathcal{M}$ and $\eta$.

3. For sources at a fixed distance the errors in the estimation of parameters are least for advanced LIGO and the highest for initial LIGO, the performance of VIRGO being in between. Although initial LIGO and VIRGO obtain similar SNRs for sources with the total mass in the range $[1,50] M_{\odot}$, the errors in VIRGO are smaller than in initial LIGO by a factor 2-4 due entirely to its greater bandwidth of observation.

4. The number of useful cycles is greater in VIRGO than LIGO for higher mass binaries $\left(M \simeq 10 M_{\odot}\right)$ but the opposite is true for lower mass binaries.

5. Parameter estimation is better if the number of useful cycles is higher but the performance also depends on the sensitivity bandwidth of the instrument. The notion of number of useful cycles together with bandwidth can be used to gauge detector performance with regard to parameter estimation.

\footnotetext{
5 The numbers listed in Tables VI and VII are those obtained by numerically integrating Eqs 4.5 and 4.6 without any further reexpansion of $\mathcal{B}_{C}$ in Eq. 4.4.
} 
6. The variation of the Fourier amplitude of the gravitational waveform across different PN orders arising from its dependence on the frequency-sweep $d F / d t$, and its implication on parameter estimation is examined. We present a Table showing how the SNR varies across the PN orders for the initial LIGO noise curve. This correction affects the errors associated with parameter estimation by less than $10 \%$ and motivates an analysis using the complete waveform including all other harmonic contributions to the GW amplitude from the 'plus' and 'cross' polarisations which are now available up to $2.5 \mathrm{PN}$ in the comparable mass case [12].

\section{B. Limitations, Caveats and Future directions}

We conclude by pointing out the regime of validity of our analysis of error bounds, its limitations and possible future directions.

1. Our estimates are based on the Cramer-Rao bound which is valid only in the regime of high SNR. Though at a SNR of 10 our calculations may be reasonably secure, in general they are less rigorous and provide only an upper bound on the errors involved. A full-fledged Monte-Carlo simulation would provide tighter bounds, though that would be computationally quite expensive.

2. In Sec IV we addressed the effect of inclusion of amplitude corrections arising from the frequency-sweep. This treatment is not fully consistent as one neglects the amplitude corrections from the other harmonics of the orbital frequency; a future study should address this issue more consistently.

3. Based on the recent runs of the GW detectors LIGO and VIRGO more 'realistic' noise curves are now available. The parameter estimation using these realistic noise curves should be eventually addressed.

4. A similar study in the case of spinning binaries is not possible until the terms corresponding to the effect of spins in the phasing formula are available beyond the present $2 \mathrm{PN}$ accuracy.

5. A more detailed study is needed for completely understanding of the reasons for PN variations of the errors. We leave this for future study.

6. The higher order phasing terms could play a major role also in the estimation of distance of the binary for a network of detectors. We will address this problem in a future work.

While finalising this paper we learnt that E. Berti and A. Buonanno have also looked at the estimation of parameters using the $3.5 \mathrm{PN}$ phasing formula [48].

\section{Acknowledgments}

We thank the LIGO Scientific Collaboration for providing us an estimate of the Advanced LIGO noise curve. We thank R. Balasubramanian, Luc Blanchet and Sanjeev Dhurandhar for useful discussions, suggestions and critical comments on the manuscript. We are grateful to Alessandra Buonanno for bringing to our notice an incorrect normalization used in the log term at 3PN in the phasing formula in an earlier version of the paper. K.G.A. would like to thank P. Ajith for useful discussions. P.A.S. thanks the Raman Research Institute for a VSR fellowship and hospitality. B.R.I. thanks the University of Wales and Cardiff, Institut d'Astrophysique de Paris and Institut des Hautes Études Scientifiques, France for hospitality during the final stages of the writing of the paper. This research was supported partly by grants from the Leverhulme Trust and the Particle Physics and Astronomy Research Council, UK. BSS thanks the Raman Research Institute, India, for supporting his visit in July-August 2004 during which some of this research was carried out. All the analytical as well as numerical calculations leading to the results of the paper have been performed with the aid of Mathematica.

[1] http://www.ligo.caltech.edu/ http://www.virgo.infn.it/http://www.geo600.uni-hannover.de/ http://tamago.mtk.nao.ac.jp/

[2] C. Cutler and K.S. Thorne, Proceedings of $16^{\text {th }}$ international conference on General relativity and Gravitation, Eds N.T. Bishop and S.D. Maharaj (World Scientific, 2002); Preprint gr-qc/0204090 (2002).

[3] S.A. Hughes, Annals Phys. 303142 (2003); Preprint astro-ph/0210481 B.S. Sathyaprakash, gr-qc/0405136 (2004).

[4] L. Blanchet, Liv. Rev. Relativ. 5, 3 (2002).

[5] C. Peters, Phys. Rev. 136, B1224 (1964). 
[6] K.S. Thorne in 300 years of gravity, edited by S.W. Hawking and W. Israel (Cambridge University Press, Cambridge, England,1987) 330.

[7] C.W. Helstrom, Statistical Theory of Signal Detection, (Pergamon, Oxford, England, 1968).

[8] B.F. Schutz, In The Detection of Gravitational Radiation, Edited by D. Blair (Cambridge University Press, England, 1989).

[9] L. Blanchet, T. Damour and B.R. Iyer, Phys. Rev. D51, 5360 (1995); 54, 1860 (E) (1996); C.M. Will and A.G. Wiseman, Phys. Rev. D 54, 4813 (1996); L. Blanchet, T. Damour, B.R. Iyer, C.M. Will and A.G. Wiseman, Phys. Rev. Lett. 74, 3515 (1995); L. Blanchet, Phys. Rev. D 54, 1417 (1996); L. Blanchet, Class. Quant. Grav. 15, 113 (1998); L. Blanchet, B.R. Iyer and B. Joguet, Phys. Rev. D 65, 064005 (2002).

[10] L. Blanchet, G. Faye, B.R. Iyer and B. Joguet, Phys. Rev. D 65, 061501(R) (2002);

[11] L. Blanchet, B.R. Iyer, C.M. Will and A.G. Wiseman, Class. Quant. Grav. 13, 575 (1996)

[12] K.G. Arun, L. Blanchet, B.R. Iyer and M.S.S. Qusailah, Class. Quant. Grav. 21, 3771 (2004); Preprint gr-qc/0404085

[13] A.M. Sintes and A. Vecchio, gr-qc/0005058 (2000); and gr-qc/0005059(2000).

[14] R.W. Hellings and T.A. Moore, Class. Quant. Grav. 20, S181 (2002)

[15] T.A. Moore and R.W. Hellings, Phys. Rev. D 65, 062001 (2003).

[16] C.R. Rao, Bullet. Calcutta Math. Soc. 37, 81 (1945); H. Cramer, Mathematical methods in statistics (Princeton University Press, NJ, 1946)

[17] K.D. Kokkotas, A. Królak and G. Tsegas, Class. Quant. Grav. 11, 1901 (1994).

[18] R. Balasubramanian, B.S. Sathyaprakash and S.V. Dhurandhar, Phys. Rev. D 53, 3033 (1996); 54, 1860 (E) (1996) .

[19] R. Balasubramanian, B.S. Sathyaprakash and S.V. Dhurandhar, Pramana 45, L463 (1995)

[20] R. Balasubramanian and S.V. Dhurandhar, Phys. Rev. D 57, 3408 (1998).

[21] D. Nicholson and A. Vecchio, Phys. Rev. D 57,4588 (1998).

[22] C. Cutler and E.E. Flanagan, Phys. Rev. D 49, 2658 (1994).

[23] P. Jaranowski and A. Królak, Phys. Rev. D 49, 1723 (1994).

[24] P. Jaranowski, K.D. Kokkotas, A. Królak and G. Tsegas, Class. Quant. Grav. 13, 1279 (1996).

[25] E. Poisson and C.M. Will , Phys. Rev.D 52, 848 (1995).

[26] A. Królak, K.D. Kokkotas and G. Schäfer, Phys. Rev. D 52, 2089 (1995).

[27] E. Berti, A. Buonanno and C.M. Will, gr-qc/0411129

[28] T. Damour, P. Jaranowski and G. Schäfer, Phys. Lett. B 513, 147 (2001).

[29] L. Blanchet, T. Damour and G Esposito-Farése, Phys. Rev. D 69, 124007 (2004).

[30] L. Blanchet, T. Damour, G. Esposito-Farèse and B.R. Iyer, Phys. Rev. Lett. 93, 091101 (2004).

[31] L. Blanchet and B.R. Iyer, Phys. Rev. D 71, 024004 (2005); gr-qc/0409094

[32] L. Blanchet, T. Damour and B.R. Iyer, Class. Quant. Grav. 22, 155 (2005); gr-qc/0410021

[33] L. Blanchet, T. Damour, G. Esposito-Farèse and B.R. Iyer (2005); gr-qc/0503044

[34] Y. Itoh and T. Futamase, Phys. Rev. D 68, 121501(R) (2003); Y. Itoh, Phys. Rev. D 69, 064018 (2004)

[35] T. Damour, B.R. Iyer and B.S. Sathyaprakash, Phys. Rev. D 63, 044023 (2001).

[36] L. E. Kidder, Phys. Rev. D 52, 821 (1995); L.E. Kidder, C.M. Will and A.G. Wiseman, Phys. Rev. D 47, R4183 (1993).

[37] T. Damour, B.R. Iyer and B.S. Sathyaprakash, Phys. Rev.D 62, 084036 (2000).

[38] K.S. Thorne in [6]; S.V. Dhurandhar, A. Królak, B.F. Schutz and J. Watkins (unpublished); B.S. Sathyaprakash, Phys. Rev. D 50, R7111 (1994); S. Droz, D.J. Knapp, E. Poisson and B.J. Owen, Phys. Rev. D 59, 124016 (1999).

[39] L.S. Finn, Phys. Rev. D 46, 5236 (1992).

[40] L.S. Finn and D.F. Chernoff, Phys. Rev. D 47, 2198 (1993).

[41] L. Blanchet and B.S. Sathyaprakash, Class. Quant. Grav. 11, 2807 (1994).

[42] L.A. Wainstein and V.D. Zubakov, Extraction of Signals from Noise, (Prentice-Hall, Englewood Cliffs, 1962).

[43] A. Królak, J.A. Lobo and B.J. Meers, Phys. Rev. D 48, 3451 (1993).

[44] M.H.A. Davies, in Gravitational Wave Data Analysis, edited by B.F. Schutz (Kluwer Academic, Dordrecht, 1989).

[45] T. Damour, B.R. Iyer and B.S. Sathyaprakash, Phys. Rev. D 66, 027502 (2002).

[46] A. Abramovici et. al., Science 256, 325 (1992).

[47] P. Ajith, B.R. Iyer, C.A.K. Robinson and B.S. Sathyaprakash, Phys. Rev. D 71, 044029 (2005).

[48] E. Berti and A. Buonanno (In preparation) 